\title{
Population norms for the EQ-5D-3L in China derived from the 2013 National Health Services Survey
}

Qiang Yao ${ }^{1,2}$, Chaojie Liu ${ }^{2}$ Yaoguang Zhang ${ }^{3}$, Ling $\mathrm{Xu}^{4}$

${ }^{1}$ School of Political Science and Public Administration, Wuhan University, Wuhan, Hubei, China

${ }^{2}$ School of Psychology and Public Health, La Trobe University, Melbourne, VIC, Australia

${ }^{3}$ Centre for Health Statistics Information, National Health Commission, Beijing, China

${ }^{4}$ China Health Human Resources, National Health Commission, Beijing, China

\section{Correspondence to:}

\section{Yaoguang Zhang}

Centre for Health Statistics Information National Health Commission

A38 Beilishi Road

Xicheng District

Beijing

China

zhangyg@nhc.gov.cn

\section{Ling $\mathrm{Xu}$}

China Health Human Resources

National Health Commission

14 Zhichun Road

Haidian District

Beijing

China

xuling@nhc.gov.cn
Background EQ-5D-3L is one of the most commonly used instruments for assessing health-related quality of life and cost-utility analyses, but it is not yet available in China. This study aims to develop population norms for the EQ-5D-3L in China in order to encourage appropriate use and interpretation of the EQ-5D-3L instrument.

Methods Data were extracted from the 2013 National Health Services Survey on a nationally representative sample of 188720 participants. The utility index based on the 2018 Chinese preference-based value sets were calculated for the participants with different demographic and socio-economic characteristics. Differences in reported problems and visual analogue scale (VAS) and utility index scores were tested using a logistic, linear and tobit regression model, respectively.

Results The Chinese respondents were less likely to report problems on the EQ-5D dimensions compared with most populations in other countries. Pain/discomfort was the most commonly reported problem (12.6\%). This resulted in a high ceiling effect $(84.19 \%)$ on the utility index and high mean scores for the utility index $(0.985 \pm 0.056)$ and VAS $(80.91 \pm 13.74)$ in the Chinese population. Those who were younger, better educated, employed, married, had no illness condition, lived in a more developed region and had a higher income obtained higher scores in both VAS and utility index. The VAS and utility index scores were also associated with gender, residency and lifestyles, but not always in a consistent way. Male and rural residents had a higher VAS score but not in the utility index compared with their female and urban counterparts.

Conclusions This study provides national population norms for the EQ-5D-3L based on the 2018 Chinese preference-based value sets. The norms can be used as a reference for health evaluation studies. Cautions need to be taken for presenting and interpreting the utility index results given the high ceiling effect of the EQ5D-3L instrument.

Health-related quality of life (HRQoL) measures the perceived well-being of people in terms of their physical, mental, social and spiritual functioning [1,2]. It adopts a philosophy of people-centred integrated care, which has attracted increasing attention around the world. HRQoL is now widely used in assessing health outcomes of both individual (eg, medical procedure) and population (eg, policy) interventions [3,4].

However, like any other health indicators, population norms are required to interpret the implications of the HRQOL measurements. They could provide a reference for tracking 
and comparisons [5,6], which is particularly important when there is no control group. Population norms can help define what is deemed normal or abnormal in different cultures. It is important to note that population norms may also change over time [7-9]. For HRQoL, this could be an indication of changes in population values on health or their health status or both. Therefore, the interpretation of HRQoL results has to be anchored in the historical and cultural contexts of the assessed population.

The EQ-5D, developed by the EuroQol Group in the 1990s, is one of the most commonly used instruments for assessing HRQoL. It is a simple and psychometrically sound instrument available in more than 170 languages [10]. The EQ-5D has three versions: EQ-5D-3L, EQ-5D-5L, and EQ-5D-Y. The former two were designed for adult populations and the latter one for children and adolescents aged 7 to 12 years. The EQ-5D instrument consists of a descriptive system and a self-report visual analogue scale (VAS). The descriptive system contains five items measuring the dimension of mobility, self-care, usual activities, pain/discomfort and anxiety/depression, respectively. Each dimension is assessed using either a three-point (no, moderate, severe) scale (EQ-5D3L) or a five-point (no, slight, moderate, severe, extreme) scale (EQ-5D-5L). The combination of health states in relation to the five dimensions can be converted into a single summary index value (also known as utility index) in line with the health preference from the general population: a utility index 0 indicates death while 1 indicates full health. The VAS records self-rated general health on a vertical visual analogue scale ranging from 'Worst imaginable health state' (0) to 'Best imaginable health state' (100) [11,12]. The utility index of individuals or subpopulations needs to be examined against their population norms. More than 30 countries have subsequently established national population norms for the EQ-5D [3,4,6,7,13-26]. These norms have been successfully used in health economic evaluation and other patient-reported outcome-based studies [24].

The EuroQol Group provided population norms for the EQ-5D-3L utility index in China based on a small sample size $(n=8031)$ using a scoring algorithm derived from the European preference-based value sets $[27,28]$. However, some researchers expressed concerns about reporting the EQ-5D-3L results based on value sets derived from other countries [29]. For example, Clemens and colleagues noted the differences in the EQ-5D utility index based on the value sets derived from the preference of the local Australian population in comparison with those resulting from the UK and the US value sets [16]. Such differences may be even more profound given the greater cultural difference between China and the European countries. Indeed, Wu and colleagues identified significant differences in the utility index for some health states between the results based on the Chinese preference value sets and those derived from the UK and Japanese populations [30]. Sun and colleagues [31] reported problems in the five dimensions of the EQ-5D-3L without converting the results into utility index using the European value sets as recommended by Janssen and colleagues [27]. A large number of countries have established their own national value sets [32].

The EQ-5D-3L has been validated in a range of Chinese populations [33,34], including in the general public [31] and those with disease conditions such as hypertension [35], diabetes [36], cancer [37], and heart disease [29]. Since 2008, the EQ-5D-3L instrument has been included in the National Health Services Survey (NHSS) in China [38]. It has been recommended as a tool for conducting health technology assessment in China [29]. In 2014, Liu GG et al published the EQ-5D-3L value sets derived from a sample of urban Chinese populations [29], which prompted the development of local population norms at several provinces in China [35,39]. But Liu's value sets suffered from a serious bias toward big cities [40]. Significant urban-rural differences in public preferences on health exist even after controlling for variations in socioeconomic status [31]. In 2018, a new version of the value sets was made available by Zhuo L et al using a national representative sample [41]. This enabled us to establish the national population norms for the EQ-5D-3L in China, which are essential for the appropriate use and interpretation of the EQ-5D instrument.

\section{DATA AND METHODS}

\section{Study design and data collection}

A cross-sectional survey was conducted using the EQ-5D-3L in China. Data were obtained from the 5th National Health Services Survey undertaken in September 2013 [42]. The NHSS has been the largest national representative household survey organised by the Centre for Health Statistics and Information of the Ministry of Health in China every five years since 1993. The NHHS followed a robust design and strict protocol. Data collected in the NHSS have been widely used in health services research and policy decision making [43-46]. The 5th NHSS adopted a four-stage stratified cluster random sampling strategy in selecting 93600 households from 1560 communities/villages, covering 780 sub-districts/townships in 156 cities/counties representing the 31 provinces in mainland China. Details on the sampling procedure used in the NHSS have been reported elsewhere $[43,45]$. 
Each household member was interviewed face-to-face separately by a trained local medical worker, tapping into the demographic and socioeconomic characteristics, lifestyle and behaviors, self-reported health, and the use of health care services of the respondents. The quality of the returned questionnaire data was checked by the survey supervisors through a repeated survey on $5 \%$ of the participating households, which resulted in a $97.7 \%$ consistency. The sample was proven to be representative

National health service survey $(n=273,688)$

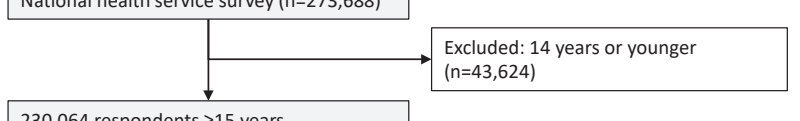

230,064 respondents $\geq 15$ years

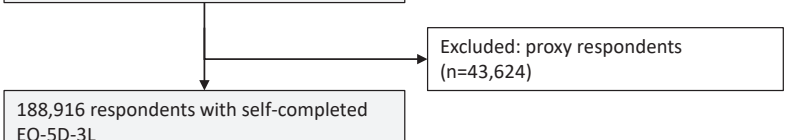

EQ-5D-3L

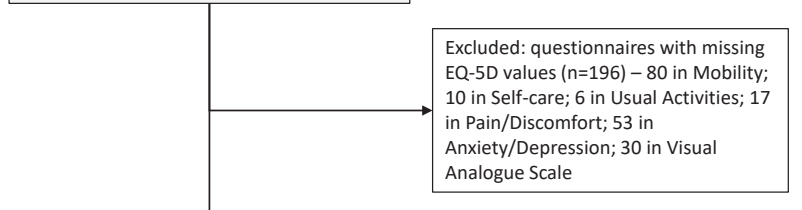

Final sample of 188,720 for data analyses

Figure 1. Identification of eligible participants for this study. of the national population without age bias as indicated by the Myer's index (2.55), DELTA dissimilarity coefficient (0.085) and GINI concentration ratio (0.0525) [42].

The EQ-5D-3L applied to those who were 15 years and older in line with other recent studies $[15,35,47]$. A total of 273688 respondents completed the questionnaire, including 230064 who were eligible for the EQ-5D-3L survey. In this study, we followed the Europe EQ-5D-3L User Guide [48] and excluded the returned questionnaires completed by a proxy respondent (a maximum of 30\% of proxy responses was allowed in the NNSS for the household members who were absent at the time of the survey). We further excluded a very small number (196) of returned questionnaires containing missing values in the EQ-5D-3L. This resulted in a final sample size of 188720 (82\% of eligible participants) for data analyses (Figure 1).

\section{Measurements}

We presented population norms for the EQ-5D-3L by gender and age. Given the large regional disparities in social and health development, the populations were further divided into eastern developed, western under-developed, and central regions in between, as well as urban vs rural.

The population norms were described using three indicators: percentage of reported problems on the five dimensions of the EQ-5D-3L, utility index, and VAS scores. The combinations of reported problems generated 243 possible health states. Each health state was assigned a value (utility index, ranging from 0.170 to 1.000) according to the nationally representative Chinese value sets developed by Zhuo and colleagues [41]. The Chinese value sets were derived from public preferences using the time-trade-off (TTO) technique as recommended by the EuroQol Group [41]. The VAS score is an indication of overall health perceived by an individual. The respondents were asked to rate their health along a scale ranging from 0 (worst health) to 100 (full health).

Factors associated with the EQ-5D-3L results were identified in line with the social determinants of health model proposed by the World Health Organisation [49]. Previous studies suggest that apart from age and gender, residency, education, employment, marital status, household income, illness conditions, lifestyle and behaviors are also significant predictors of HRQoL in China [31]. In this study, household income was categorised into quintiles according to the income distributions of the local cities or counties. Illness conditions were captured by reported acute conditions over the two weeks prior to the survey (yes or no), chronic conditions diagnosed by a doctor during the previous six months (yes or no), and episodes of hospital care over the previous 12 months (yes or no). Lifestyle and behaviors were measured by the current status of smoking (yes or no), drinking (yes or no during the last 12 months), and weekly physical exercise (yes or no over the last 6 months). Further details about the definitions of these variables can be found in the NHSS guidelines [42].

\section{Statistical analysis}

We calculated the percentage of respondents reporting problems on each of the five dimensions of the EQ-5D$3 \mathrm{~L}$, as well as the percentage of respondents who reported problems in any dimension. Population differences in relation to the reported problems were tested using Pearson $\chi^{2}$ tests. Multivariate binary logistic regression models (with or without problems) were performed to identify the factors associated with the reported problems.

Means with standard deviations (SD) and medians with interquartile ranges (IQR) of the utility index and VAS scores of the EQ-5D-3L were calculated in line with those of previous studies $[4,15,24,27,47]$. Population differences in the utility index and VAS scores were tested using student $t$ tests or analysis of variance (ANOVA), and Wilcoxon or Kruskal-Wallis rank sum tests. Multivariate linear regression models and Tobit regression models were performed to identify the factors associated with the VAS and the utility index scores, respectively. The Tobit approach was recommended by Zhang for censored or bounded data $[35,50]$. 
The significance level of the statistical analyses was set at 0.05 . All statistical analyses were performed using STATA version 14.0 (SE) for Windows (StataCorp LLC, College Station, TX, USA). An enter approach was adopted in the regression modelling, with all of the independent variables being coded as categorical variables and compared with a reference group. Given that the statistical significance can simply be a function of large sample size, we also used the Cohen effect size (average difference in the score divided by the standard deviation of the score in the group for comparison) [51,52] to judge the significance of the differences of the utility index and VAS scores. According to Cohen, a size below 0.2 indicates a small effect, while 0.5 and 0.8 indicate a medium and a large effect size, respectively. A medium effect size (0.5) is usually considered as a difference with clinical meaning [52].

\section{Ethics}

The present study is a secondary analysis of the NHSS 2013 data. The NHSS obtained ethics approval from the institutional review board of the Chinese National Bureau of Statistics (license number 2013-65). Informed consent was obtained from all the respondents prior to the survey. All procedures performed in the study were in accordance with the ethical standards of the Chinese National Bureau of Statistics and with the 1975 Helsinki declaration.

\section{RESULTS}

\section{Characteristics of respondents}

Slightly more than half of the respondents (52.4\%) were women. The distribution of respondents in terms of gender, region, education, employment and marital status resembled those of the national population structure [42]. However, this study sample contained a higher proportion of respondents aged 65 years and older (17.84\%) compared with the national average 11.6\% [53] (Table 1).

Table 1. EQ-5D-3L VAS and utility index scores of respondents by socio-demographic characteristics

\begin{tabular}{|c|c|c|c|c|c|c|c|c|c|c|c|}
\hline \multirow{2}{*}{$\begin{array}{l}\text { CHARACTERISTICS OF } \\
\text { RESPONDENTS }\end{array}$} & \multirow{2}{*}{ N } & \multirow{2}{*}{$\%$} & \multicolumn{4}{|c|}{ VAS SCORE } & \multicolumn{4}{|c|}{ UTILITY INDEX } & \multirow{2}{*}{$\begin{array}{l}\text { CEILING } \\
\text { EFFECT } \\
(\%)\end{array}$} \\
\hline & & & Mean & SD & $\mathrm{t} / \mathrm{F}$ & $P$ value & Mean & SD & $\mathrm{t} / \mathrm{F}$ & $P$ value & \\
\hline \multicolumn{12}{|l|}{ Demographic } \\
\hline Gender: & & & & & 19.52 & $<0.001$ & & & 5.86 & $<0.001$ & \\
\hline Male & 89830 & 47.60 & 81.56 & 13.53 & & & 0.986 & 0.056 & & & 85.73 \\
\hline Female & 98890 & 52.40 & 80.32 & 13.91 & & & 0.984 & 0.056 & & & 82.79 \\
\hline Age (in years): & & & & & 7030.70 & $<0.001$ & & & 2752.71 & $<0.001$ & \\
\hline $15-24$ & 14094 & 7.47 & 90.40 & 8.35 & & & 0.998 & 0.023 & & & 97.98 \\
\hline $25-34$ & 24347 & 12.90 & 88.01 & 9.39 & & & 0.997 & 0.024 & & & 96.37 \\
\hline $35-44$ & 35081 & 18.59 & 84.68 & 11.34 & & & 0.995 & 0.031 & & & 92.07 \\
\hline $45-54$ & 41000 & 21.73 & 81.35 & 12.82 & & & 0.990 & 0.042 & & & 87.03 \\
\hline $55-64$ & 40532 & 21.48 & 77.29 & 13.56 & & & 0.983 & 0.056 & & & 79.03 \\
\hline $65-74$ & 22138 & 11.73 & 73.08 & 14.43 & & & 0.969 & 0.077 & & & 69.47 \\
\hline $75+$ & 11528 & 6.11 & 69.05 & 15.32 & & & 0.933 & 0.119 & & & 53.91 \\
\hline \multicolumn{12}{|l|}{ Location } \\
\hline Residency: & & & & & 100.06 & $<0.001$ & & & 15.12 & $<0.001$ & \\
\hline Urban & 94064 & 49.84 & 80.59 & 13.78 & & & 0.985 & 0.055 & & & 84.16 \\
\hline Rural & 94656 & 50.16 & 81.23 & 13.71 & & & 0.984 & 0.058 & & & 84.22 \\
\hline Region: & & & & & 435.23 & $<0.001$ & & & 40.53 & $<0.001$ & \\
\hline Eastern & 66575 & 35.28 & 82.12 & 13.25 & & & 0.986 & 0.054 & & & 85.83 \\
\hline Central & 58306 & 30.90 & 80.59 & 13.99 & & & 0.984 & 0.058 & & & 83.57 \\
\hline Western & 63839 & 33.83 & 79.94 & 13.94 & & & 0.984 & 0.057 & & & 83.04 \\
\hline \multicolumn{12}{|l|}{ Socio-economic } \\
\hline Educational attainment: & & & & & 3743.86 & $<0.001$ & & & 1542.77 & $<0.001$ & \\
\hline Illiterate & 22709 & 12.03 & 73.13 & 15.40 & & & 0.961 & 0.091 & & & 67.36 \\
\hline Primary school & 48953 & 25.94 & 78.23 & 14.12 & & & 0.980 & 0.063 & & & 79.06 \\
\hline Junior middle school & 65877 & 34.91 & 83.05 & 12.59 & & & 0.990 & 0.044 & & & 88.49 \\
\hline Senior middle school & 32435 & 17.19 & 83.44 & 12.52 & & & 0.992 & 0.041 & & & 89.90 \\
\hline University/college or above & 18746 & 9.93 & 85.44 & 11.22 & & & 0.995 & 0.028 & & & 92.94 \\
\hline
\end{tabular}


Table 1. Continued

\begin{tabular}{|c|c|c|c|c|c|c|c|c|c|c|c|}
\hline \multirow{2}{*}{$\begin{array}{l}\text { CHARACTERISTICS OF } \\
\text { RESPONDENTS }\end{array}$} & \multirow{2}{*}{$\mathbf{N}$} & \multirow{2}{*}{$\%$} & \multicolumn{4}{|c|}{ VAS SCORE } & \multicolumn{4}{|c|}{ UTILITY INDEX } & \multirow{2}{*}{$\begin{array}{c}\text { CEILING } \\
\text { EFFECT } \\
(\%)\end{array}$} \\
\hline & & & Mean & SD & $\mathrm{t} / \mathrm{F}$ & $P$ value & Mean & SD & $\mathrm{t} / \mathrm{F}$ & $P$ value & \\
\hline $\begin{array}{l}\text { Local ranking of average } \\
\text { household income: }\end{array}$ & & & & & 712.90 & $<0.001$ & & & 360.76 & $<0.001$ & \\
\hline Lowest (<percentile 20) & 35702 & 18.93 & 77.69 & 15.56 & & & 0.975 & 0.072 & & & 76.90 \\
\hline Low (percentile 20-39) & 35471 & 18.80 & 80.53 & 13.86 & & & 0.984 & 0.058 & & & 83.57 \\
\hline Middle (percentile 40-59) & 37124 & 19.68 & 81.63 & 13.26 & & & 0.987 & 0.052 & & & 85.81 \\
\hline High (percentile 60-79) & 39084 & 20.72 & 81.92 & 12.94 & & & 0.988 & 0.049 & & & 86.61 \\
\hline Highest ( $\geq$ percentile 80 ) & 41251 & 21.87 & 82.43 & 12.63 & & & 0.989 & 0.047 & & & 87.30 \\
\hline Employment: & & & & & 5374.26 & $<0.001$ & & & 2812.74 & $<0.001$ & \\
\hline Employed & 127614 & 67.62 & 83.00 & 12.34 & & & 0.992 & 0.035 & & & 88.65 \\
\hline Retired & 27274 & 14.45 & 75.67 & 13.79 & & & 0.975 & 0.072 & & & 75.96 \\
\hline Student & 4747 & 2.52 & 91.05 & 8.08 & & & 0.999 & 0.016 & & & 98.42 \\
\hline Unemployed & 29085 & 15.41 & 75.01 & 16.49 & & & 0.961 & 0.096 & & & 70.00 \\
\hline Marital status: & & & & & 3757.16 & $<0.001$ & & & 1623.95 & $<0.001$ & \\
\hline Never married/Single & 17131 & 9.08 & 88.10 & 11.27 & & & 0.993 & 0.044 & & & 94.12 \\
\hline Married & 155755 & 82.53 & 80.92 & 13.39 & & & 0.987 & 0.052 & & & 85.05 \\
\hline Widowed & 12932 & 6.85 & 71.61 & 15.06 & & & 0.953 & 0.097 & & & 61.32 \\
\hline Divorced & 2898 & 1.54 & 79.60 & 14.71 & & & 0.982 & 0.061 & & & 81.44 \\
\hline \multicolumn{12}{|l|}{ Illness condition } \\
\hline Two-week morbidity: & & & & & -130.00 & $<0.001$ & & & -67.82 & $<0.001$ & \\
\hline Yes & 44986 & 23.84 & 72.74 & 15.57 & & & 0.963 & 0.088 & & & 65.28 \\
\hline No & 143734 & 76.16 & 83.47 & 12.03 & & & 0.992 & 0.039 & & & 90.10 \\
\hline Chronic disease: & & & & & -160.00 & $<0.001$ & & & -75.39 & $<0.001$ & \\
\hline Yes & 50698 & 26.86 & 72.37 & 15.34 & & & 0.963 & 0.087 & & & 65.16 \\
\hline No & 138022 & 73.14 & 84.05 & 11.63 & & & 0.993 & 0.036 & & & 91.18 \\
\hline One-year hospital admission: & & & & & -64.53 & $<0.001$ & & & -39.93 & $<0.001$ & \\
\hline Yes & 17016 & 9.02 & 73.09 & 16.88 & & & 0.957 & 0.100 & & & 65.02 \\
\hline No & 171681 & 90.98 & 81.69 & 13.14 & & & 0.988 & 0.049 & & & 86.09 \\
\hline \multicolumn{12}{|c|}{ Lifestyle and behaviors } \\
\hline Smoking: & & & & & 13.41 & $<0.001$ & & & 18.42 & $<0.001$ & \\
\hline Yes & 49208 & 26.09 & 81.60 & 13.05 & & & 0.988 & 0.045 & & & 86.21 \\
\hline No & 139399 & 73.91 & 80.67 & 13.97 & & & 0.984 & 0.060 & & & 83.47 \\
\hline Drinking: & & & & & 25.42 & $<0.001$ & & & 31.15 & $<0.001$ & \\
\hline Yes & 44005 & 23.32 & 82.28 & 12.46 & & & 0.991 & 0.037 & & & 86.95 \\
\hline No & 144706 & 76.68 & 80.50 & 14.09 & & & 0.983 & 0.061 & & & 83.35 \\
\hline Physical exercise: & & & & & 0.71 & 0.477 & & & 25.14 & $<0.001$ & \\
\hline Yes & 55843 & 29.67 & 80.94 & 13.10 & & & 0.989 & 0.038 & & & 85.10 \\
\hline No & 132372 & 70.33 & 80.90 & 14.01 & & & 0.983 & 0.062 & & & 83.79 \\
\hline Total & 188720 & 100.00 & 80.91 & 13.74 & - & - & 0.985 & 0.056 & - & - & 84.19 \\
\hline
\end{tabular}

VAS - visual analogue scale

\section{Percentage of reported health problems}

Pain/discomfort was the most frequently reported problem (12.6\%), followed by problems in mobility (5.9\%) and anxiety/depression (5.3\%). The least reported problem was in self-care (3.1\%) (Table 2). Women were more likely to report problems than men in relation to pain/discomfort and anxiety/depression, but less in other dimensions of the EQ-5D. The proportion of respondents reporting problems increased with age. Socioeconomic gradients were evident, and those with a higher socio-economic status (better educated, higher income and employed) were less likely to report problems. Rural residents and those residing in the less developed central and western regions were more likely to report problems on all of the five dimensions than others. Those who did not smoke or drink reported more problems than those who did (Table 3).

\section{EQ-5D-3L utility index and VAS score}

The distribution of the utility index was negatively skewed (Figure 2). The respondents reported 161 health states out of a possible 243 . More than $84 \%$ of respondents had a full health state (" 1111 "), followed by the 
Table 2. Percentage (\%) of respondents reporting problems in the five dimensions of EQ-5D-3L

\begin{tabular}{|c|c|c|c|c|c|c|c|c|c|c|c|c|c|c|c|c|}
\hline \multirow[b]{2}{*}{ VARIABLE } & \multirow[b]{2}{*}{$\begin{array}{l}\text { ANY } \\
\text { PROB- } \\
\text { LEMS }\end{array}$} & \multicolumn{3}{|c|}{ MOBILITY } & \multicolumn{3}{|c|}{ SELF-CARE } & \multicolumn{3}{|c|}{ USUAL ACTIVITIES } & \multicolumn{3}{|c|}{ PAIN/DISCOMFORT } & \multicolumn{3}{|c|}{ ANXIETY/DEPRESSION } \\
\hline & & Z & $\begin{array}{l}\text { ̋̈ } \\
\text { ஸे }\end{array}$ & 苞 & Z & छั & $\begin{array}{l}\frac{0}{0} \\
\text { స్ } \\
5\end{array}$ & ż & $\begin{array}{l}\text { ̋̃ } \\
\text { ஸे }\end{array}$ & $\begin{array}{l}\frac{u}{\frac{\pi}{\tilde{\Xi}}} \\
\frac{5}{5}\end{array}$ & ż & $\begin{array}{l}\text { ̋̃ } \\
\text { ڤ }\end{array}$ & 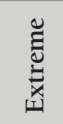 & Z & $\begin{array}{l}\text { ڤ̆ } \\
\text { ஸे }\end{array}$ & 荀 \\
\hline Gender & $P<0.001$ & \multicolumn{3}{|c|}{$P=0.008$} & \multicolumn{3}{|c|}{$P=0.029$} & \multicolumn{3}{|c|}{$P<0.001$} & \multicolumn{3}{|c|}{$P<0.001$} & \multicolumn{3}{|c|}{$P<0.001$} \\
\hline Male & 14.3 & 94.3 & 5.4 & 0.3 & 97.0 & 2.5 & 0.5 & 95.5 & 3.7 & 0.9 & 89.0 & 10.5 & 0.5 & 95.4 & 4.4 & 0.3 \\
\hline Female & 17.2 & 94.0 & 5.7 & 0.3 & 96.9 & 2.7 & 0.4 & 95.2 & 4.0 & 0.8 & 85.9 & 13.5 & 0.6 & 94.1 & 5.6 & 0.3 \\
\hline Age (in years) & $P<0.001$ & \multicolumn{3}{|c|}{$P<0.001$} & \multicolumn{3}{|c|}{$P<0.001$} & & $<0.00$ & & & $<0.00$ & & & $<0.00$ & \\
\hline $15-24$ & 2.0 & 99.5 & 0.4 & 0.1 & 99.7 & 0.3 & 0.1 & 99.5 & 0.3 & 0.1 & 98.8 & 1.1 & 0.1 & 99.1 & 0.8 & 0.1 \\
\hline $25-34$ & 3.6 & 99.3 & 0.6 & 0.1 & 99.6 & 0.4 & 0.1 & 99.4 & 0.5 & 0.1 & 97.8 & 2.1 & 0.1 & 98.4 & 1.5 & 0.1 \\
\hline $35-44$ & 7.9 & 98.5 & 1.4 & 0.1 & 99.2 & 1.3 & 0.2 & 98.7 & 1.0 & 0.2 & 94.3 & 5.6 & 0.2 & 96.6 & 3.2 & 0.2 \\
\hline $45-54$ & 13.0 & 96.9 & 3.0 & 0.2 & 98.5 & 2.6 & 0.4 & 97.5 & 2.1 & 0.4 & 89.5 & 10.1 & 0.4 & 95.3 & 4.4 & 0.2 \\
\hline 55-64 & 21.0 & 93.6 & 6.2 & 0.3 & 97.0 & 5.9 & 0.8 & 95.2 & 4.0 & 0.8 & 82.4 & 17.0 & 0.7 & 93.1 & 6.6 & 0.3 \\
\hline 65-74 & 30.5 & 86.6 & 12.8 & 0.6 & 93.2 & 14.4 & 2.8 & 89.6 & 8.8 & 1.6 & 74.7 & 24.3 & 1.0 & 91.0 & 8.6 & 0.4 \\
\hline $75+$ & 46.1 & 70.2 & 27.7 & 2.2 & 82.8 & 2.6 & 0.4 & 75.4 & 19.7 & 4.9 & 64.8 & 33.2 & 2.0 & 86.5 & 12.7 & 0.8 \\
\hline Residency & $P=0.698$ & & $=0.00$ & & & $<0.00$ & & & $<0.00$ & & & $=0.10$ & & & $<0.00$ & \\
\hline Urban & 15.8 & 94.3 & 5.4 & 0.3 & 97.2 & 2.4 & 0.4 & 95.7 & 3.5 & 0.8 & 87.6 & 12.0 & 0.5 & 95.0 & 4.7 & 0.3 \\
\hline Rural & 15.8 & 94.0 & 5.7 & 0.3 & 96.7 & 2.9 & 0.4 & 95.0 & 4.2 & 0.8 & 87.2 & 12.3 & 0.5 & 94.4 & 5.3 & 0.3 \\
\hline Region & $P<0.001$ & & $<0.00$ & & & $<0.00$ & & & $<0.00$ & & & $<0.00$ & & & $<0.00$ & \\
\hline Eastern & 14.2 & 94.6 & 5.1 & 0.3 & 97.2 & 2.4 & 0.4 & 95.8 & 3.4 & 0.8 & 88.9 & 10.7 & 0.4 & 95.8 & 4.0 & 0.2 \\
\hline Central & 16.4 & 94.0 & 5.7 & 0.3 & 96.9 & 2.6 & 0.5 & 95.4 & 3.8 & 0.8 & 86.6 & 12.8 & 0.6 & 94.5 & 5.3 & 0.3 \\
\hline Western & 17.0 & 93.8 & 5.9 & 0.3 & 96.8 & 2.9 & 0.4 & 94.9 & 4.3 & 0.8 & 86.5 & 12.9 & 0.6 & 93.8 & 5.8 & 0.4 \\
\hline Educational attainment & $P<0.001$ & & $<0.00$ & & & $<0.00$ & & & $<0.00$ & & & $<0.00$ & & & $<0.00$ & \\
\hline Illiterate & 32.6 & 84.6 & 14.5 & 1.0 & 91.1 & 7.6 & 1.3 & 86.6 & 10.9 & 2.4 & 73.4 & 25.3 & 1.3 & 88.3 & 11.1 & 0.6 \\
\hline Primary school & 20.9 & 92.0 & 7.6 & 0.4 & 96.0 & 3.5 & 0.5 & 93.9 & 5.1 & 1.0 & 83.0 & 16.4 & 0.7 & 93.4 & 6.3 & 0.3 \\
\hline Junior mi & 11.5 & 96.4 & 3.4 & 0.2 & 98.2 & 1.5 & 0.3 & 97.3 & 2.3 & 0.5 & 91.0 & 8.7 & 0.3 & 96.1 & 3.7 & 0.2 \\
\hline Senior middle school & 10.1 & 97.1 & 2.7 & 0.2 & 98.6 & 1.2 & 0.2 & 97.8 & 1.8 & 0.4 & 92.1 & 7.6 & 0.3 & 96.8 & 3.0 & 0.2 \\
\hline University/college or above & 7.1 & 98.3 & 1.6 & 0.1 & 99.2 & 0.7 & 0.1 & 98.8 & 1.0 & 0.2 & 95.0 & 4.8 & 0.1 & 97.5 & 2.4 & 0.1 \\
\hline $\begin{array}{l}\text { Local ranking of average } \\
\text { household income }\end{array}$ & $P<0.001$ & & $<0.00$ & & & $<0.00$ & & & $<0.00$ & & & $<00$ & & & 0.00 & \\
\hline Lowest $(<$ percentile 20$)$ & 23.1 & 90.2 & 9.3 & 0.5 & 94.7 & 4.6 & 0.7 & 91.9 & 6.6 & 1.5 & 81.7 & 17.4 & 0.9 & 91.6 & 7.9 & 0.5 \\
\hline Low (percentile 20-39) & 16.4 & 93.9 & 5.7 & 0.4 & 96.9 & 2.7 & 0.5 & 95.2 & 3.9 & 0.9 & 87.0 & 12.4 & 0.5 & 94.4 & 5.4 & 0.2 \\
\hline Middle (percentile 40-59) & 14.2 & 95.1 & 4.7 & 0.3 & 97.4 & 2.2 & 0.4 & 96.2 & 3.2 & 0.7 & 88.3 & 11.2 & 0.5 & 95.4 & 4.4 & 0.2 \\
\hline High (percentile 60-79) & 13.4 & 95.4 & 4.3 & 0.3 & 97.7 & 2.0 & 0.3 & 96.5 & 3.0 & 0.6 & 89.3 & 10.3 & 0.4 & 95.8 & 4.0 & 0.2 \\
\hline Highest ( $\geq$ percentile 80 ) & 12.7 & 95.7 & 4.0 & 0.2 & 97.8 & 1.9 & 0.3 & 96.7 & 2.7 & 0.6 & 90.0 & 9.7 & 0.3 & 96.2 & 3.7 & 0.2 \\
\hline Employment & $P<0.001$ & & $<0.00$ & & & $<0.00$ & & & $<0.00$ & & & $<0.00$ & & & $<0.00$ & \\
\hline Employed & 11.4 & 97.1 & 2.9 & 0.1 & 98.7 & 1.2 & 0.1 & 97.8 & 2.0 & 0.2 & 90.9 & 8.9 & 0.3 & 96.0 & 3.9 & 0.2 \\
\hline Retired & 24.0 & 89.7 & 9.7 & 0.6 & 94.8 & 4.4 & 0.9 & 92.3 & 6.1 & 1.5 & 80.9 & 18.3 & 0.8 & 94.0 & 5.7 & 0.3 \\
\hline Student & 1.6 & 99.6 & 0.3 & 0.0 & 99.8 & 0.2 & 0.0 & 99.8 & 0.2 & 0.0 & 99.2 & 0.8 & 0.1 & 99.3 & 0.6 & 0.0 \\
\hline Unemployed & 30.0 & 84.7 & 14.2 & 1.2 & 91.0 & 7.5 & 1.5 & 86.8 & 10.4 & 2.8 & 76.1 & 22.4 & 1.5 & 89.1 & 10.1 & 0.8 \\
\hline Marital status & $P<0.001$ & & $<0.00$ & & & $<0.00$ & & & $<0.00$ & & & $<0.00$ & & & $<0.00$ & \\
\hline Never married/Single & 5.9 & 97.7 & 2.1 & 0.2 & 98.5 & 1.2 & 0.3 & 97.8 & 1.7 & 0.5 & 96.3 & 3.5 & 0.3 & 97.4 & 2.4 & 0.2 \\
\hline Married & 15.0 & 95.0 & 4.8 & 0.3 & 97.5 & 2.2 & 0.4 & 96.1 & 3.2 & 0.7 & 87.9 & 11.6 & 0.5 & 95.1 & 4.7 & 0.2 \\
\hline Divorced & 38.7 & 79.7 & 19.1 & 1.1 & 88.5 & 10.0 & 1.5 & 83.4 & 13.7 & 2.8 & 69.3 & 29.4 & 1.3 & 87.4 & 12.0 & 0.7 \\
\hline Widowed & 18.6 & 94.0 & 5.7 & 0.4 & 96.7 & 2.9 & 0.4 & 95.0 & 4.1 & 0.9 & 86.2 & 13.1 & 0.7 & 91.6 & 7.7 & 0.8 \\
\hline Two-week morbidity & $P<0.001$ & & $<0.00$ & & & $<0.00$ & & & $<0.00$ & & & $<0.00$ & & & $<0.00$ & \\
\hline Yes & 34.7 & 85.6 & 13.5 & 0.9 & 92.4 & 6.4 & 1.2 & 88.4 & 9.3 & 2.3 & 70.7 & 27.9 & 1.5 & 88.3 & 11.1 & 0.7 \\
\hline No & 9.9 & 96.8 & 3.0 & 0.2 & 98.4 & 1.5 & 0.2 & 97.5 & 2.1 & 0.3 & 92.6 & 7.2 & 0.2 & 96.8 & 3.1 & 0.1 \\
\hline Chronic disease & $P<0.001$ & & $<0.00$ & & & $<0.00$ & & & $<0.00$ & & & $<0.00$ & & & $<0.00$ & \\
\hline Yes & 34.8 & 85.4 & 13.8 & 0.8 & 92.3 & 6.5 & 1.2 & 88.2 & 9.6 & 2.2 & 70.9 & 27.6 & 1.4 & 88.3 & 11.1 & 0.6 \\
\hline No & 8.8 & 97.4 & 2.5 & 0.1 & 98.6 & 1.2 & 0.2 & 98.0 & 1.7 & 0.3 & 93.4 & 6.4 & 0.2 & 97.1 & 2.8 & 0.1 \\
\hline $\begin{array}{l}\text { One-year hospital } \\
\text { admission }\end{array}$ & $P<0.001$ & & $<0.00$ & & & $<0.00$ & & & $<0.00$ & & & $<0.00$ & & & $<0.00$ & \\
\hline Yes & 35.0 & 83.4 & 15.3 & 1.3 & 90.6 & 7.7 & 1.7 & 86.0 & 11.0 & 3.1 & 70.8 & 27.2 & 2.1 & 87.0 & 12.2 & 0.9 \\
\hline No & 13.9 & 95.2 & 4.6 & 0.2 & 97.6 & 2.1 & 0.3 & 96.3 & 3.1 & 0.6 & 89.0 & 10.6 & 0.4 & 95.5 & 4.3 & 0.2 \\
\hline Smoking & $P<0.001$ & & $<0.00$ & & & $<0.00$ & & & $<0.00$ & & & $<0.00$ & & & $<0.00$ & \\
\hline Yes & 13.8 & 95.3 & 4.5 & 0.2 & 97.8 & 2.0 & 0.2 & 96.5 & 3.0 & 0.5 & 89.2 & 10.5 & 0.4 & 95.5 & 4.3 & 0.2 \\
\hline No & 16.5 & 93.7 & 5.9 & 0.4 & 96.6 & 2.9 & 0.5 & 95.0 & 4.1 & 0.9 & 86.8 & 12.7 & 0.6 & 94.5 & 5.3 & 0.3 \\
\hline
\end{tabular}


Table 2. Continued

\begin{tabular}{|c|c|c|c|c|c|c|c|c|c|c|c|c|c|c|c|c|}
\hline \multirow[b]{2}{*}{ VARIABLE } & \multirow[b]{2}{*}{$\begin{array}{l}\text { ANY } \\
\text { PROB- } \\
\text { LEMS }\end{array}$} & \multicolumn{3}{|c|}{ MOBILITY } & \multicolumn{3}{|c|}{ SELF-CARE } & \multicolumn{3}{|c|}{ USUAL ACTIVITIES } & \multicolumn{3}{|c|}{ PAIN/DISCOMFORT } & \multicolumn{3}{|c|}{ ANXIETY/DEPRESSION } \\
\hline & & ż & $\begin{array}{l}\text { हू } \\
\text { ڤ̆ }\end{array}$ & 苛 & z & 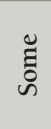 & $\begin{array}{l}\frac{0}{\pi} \\
\text { స్ } \\
\text { కే }\end{array}$ & z & 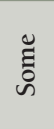 & 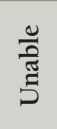 & z & $\begin{array}{l}\text { ڤ̆ } \\
\text { ڤे }\end{array}$ & 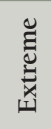 & $\dot{z}$ & ڤ̆ & 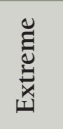 \\
\hline Drinking & $P<0.001$ & \multicolumn{3}{|c|}{$P<0.001$} & \multicolumn{3}{|c|}{$P<0.001$} & \multicolumn{3}{|c|}{$P<0.001$} & \multicolumn{3}{|c|}{$P<0.001$} & \multicolumn{3}{|c|}{$P<0.001$} \\
\hline Yes & 13.1 & 96.2 & 3.7 & 0.1 & 98.5 & 1.4 & 0.1 & 97.5 & 2.3 & 0.3 & 89.7 & 10.0 & 0.2 & 96.0 & 3.9 & 0.2 \\
\hline No & 16.7 & 93.5 & 6.1 & 0.4 & 96.5 & 3.0 & 0.5 & 94.7 & 4.3 & 1.0 & 86.7 & 12.7 & 0.6 & 94.4 & 5.4 & 0.3 \\
\hline Physical exercise & $P<0.001$ & \multicolumn{3}{|c|}{$P<0.001$} & \multicolumn{3}{|c|}{$P<0.001$} & \multicolumn{3}{|c|}{$P<0.001$} & \multicolumn{3}{|c|}{$P<0.001$} & \multicolumn{3}{|c|}{$P<0.001$} \\
\hline Yes & 14.9 & 95.5 & 4.4 & 0.1 & 98.1 & 1.8 & 0.1 & 96.8 & 2.9 & 0.3 & 88.1 & 11.6 & 0.3 & 95.7 & 4.1 & 0.1 \\
\hline No & 16.2 & 93.6 & 6.0 & 0.4 & 96.5 & 3.0 & 0.6 & 94.8 & 4.2 & 1.0 & 87.1 & 12.3 & 0.6 & 94.3 & 5.4 & 0.3 \\
\hline Total & 15.8 & 94.1 & 5.5 & 0.3 & 97.0 & 2.6 & 0.4 & 95.4 & 3.8 & 0.8 & 87.4 & 12.1 & 0.5 & 94.7 & 5.0 & 0.3 \\
\hline
\end{tabular}

Table 3. Factors associated with the percentage of problems reported by respondents in the five EQ-5D dimensions - results of logistic regression models

\begin{tabular}{|c|c|c|c|c|c|c|c|c|c|c|c|c|c|c|c|}
\hline \multirow[b]{2}{*}{ VARIABLE } & \multicolumn{3}{|c|}{ MOBILITY } & \multicolumn{3}{|c|}{ SELF-CARE } & \multicolumn{3}{|c|}{ USUAL ACTIVITIES } & \multicolumn{3}{|c|}{ PAIN/DISCOMFORT } & \multicolumn{3}{|c|}{ ANXIETY/DEPRESSION } \\
\hline & $\mathrm{AOR}^{*}$ & $\begin{array}{c}95 \% \\
\text { CI }\end{array}$ & $P$ & AOR & $\begin{array}{c}95 \% \\
\text { CI }\end{array}$ & $\mathbf{P}$ & AOR & $\begin{array}{l}(95 \% \\
\text { CI }\end{array}$ & $\mathrm{P}$ & AOR & $\begin{array}{l}(95 \% \\
\mathrm{CI})\end{array}$ & $\mathbf{P}$ & AOR & $\begin{array}{l}(95 \% \\
\mathrm{CI})\end{array}$ & $\mathrm{P}$ \\
\hline \multicolumn{16}{|l|}{ Gender } \\
\hline \multicolumn{16}{|l|}{ Male (reference) } \\
\hline Female & 0.727 & $\begin{array}{l}0.688 \\
0.768 \\
\end{array}$ & $<0.001$ & 0.615 & $\begin{array}{l}0.573 \\
0.661 \\
\end{array}$ & $<0.001$ & 0.652 & $\begin{array}{l}0.613 \\
0.692 \\
\end{array}$ & $<0.001$ & 1.196 & $\begin{array}{l}1.149 \\
1.245 \\
\end{array}$ & $<0.001$ & 1.064 & $\begin{array}{l}1.006 \\
1.124 \\
\end{array}$ & 0.030 \\
\hline \multicolumn{16}{|l|}{ Age in years } \\
\hline \multicolumn{16}{|l|}{ 15-24 (reference) } \\
\hline $25-34$ & 1.818 & $\begin{array}{l}1.308 \\
2.525\end{array}$ & $<0.001$ & 1.806 & $\begin{array}{l}1.233 \\
2.646\end{array}$ & 0.002 & 1.839 & $\begin{array}{l}1.333 \\
2.537\end{array}$ & $<0.001$ & 1.717 & $\begin{array}{l}1.417 \\
2.082\end{array}$ & $<0.001$ & 2.225 & $\begin{array}{l}1.771 \\
2.795\end{array}$ & $<0.001$ \\
\hline $35-44$ & 3.754 & $\begin{array}{l}2.749 \\
5.126\end{array}$ & $<0.001$ & 3.413 & $\begin{array}{l}2.378 \\
4.898\end{array}$ & $<0.001$ & 3.567 & $\begin{array}{l}2.627 \\
4.842\end{array}$ & $<0.001$ & 3.958 & $\begin{array}{l}3.299 \\
4.750\end{array}$ & $<0.001$ & 4.505 & $\begin{array}{l}3.611 \\
5.621\end{array}$ & $<0.001$ \\
\hline $45-54$ & 6.582 & $\begin{array}{l}4.847 \\
8.939 \\
\end{array}$ & $<0.001$ & 5.124 & $\begin{array}{l}3.602 \\
7.287\end{array}$ & $<0.001$ & 5.699 & $\begin{array}{l}4.221 \\
7.693\end{array}$ & $<0.001$ & 6.296 & $\begin{array}{l}5.253 \\
7.546\end{array}$ & $<0.001$ & 5.315 & $\begin{array}{l}4.261 \\
6.631 \\
\end{array}$ & $<0.001$ \\
\hline $55-64$ & 9.276 & $\begin{array}{l}6.829 \\
12.601\end{array}$ & $<0.001$ & 6.454 & $\begin{array}{l}4.535 \\
9.187\end{array}$ & $<0.001$ & 7.042 & $\begin{array}{l}5.211 \\
9.515\end{array}$ & $<0.001$ & 8.369 & $\begin{array}{l}6.973 \\
10.045 \\
\end{array}$ & $<0.001$ & 5.947 & $\begin{array}{l}4.758 \\
7.435\end{array}$ & $<0.001$ \\
\hline $65-74$ & 14.618 & $\begin{array}{l}10.734 \\
19.908 \\
\end{array}$ & $<0.001$ & 9.737 & $\begin{array}{l}6.820 \\
13.903 \\
\end{array}$ & $<0.001$ & 10.863 & $\begin{array}{l}8.013 \\
14.725 \\
\end{array}$ & $<0.001$ & 10.229 & $\begin{array}{l}8.498 \\
12.313 \\
\end{array}$ & $<0.001$ & 5.905 & $\begin{array}{l}4.700 \\
7.418 \\
\end{array}$ & $<0.001$ \\
\hline $75+$ & 31.140 & $\begin{array}{l}22.803 \\
42.526 \\
\end{array}$ & $<0.001$ & 19.503 & $\begin{array}{l}13.616 \\
27.936 \\
\end{array}$ & $<0.001$ & 22.489 & $\begin{array}{l}16.541 \\
30.577\end{array}$ & $<0.001$ & 13.617 & $\begin{array}{l}11.262 \\
16.465 \\
\end{array}$ & $<0.001$ & 7.359 & $\begin{array}{l}5.823 \\
9.300 \\
\end{array}$ & $<0.001$ \\
\hline \multicolumn{16}{|l|}{ Location } \\
\hline \multicolumn{16}{|l|}{ Urban (reference) } \\
\hline Rural & 1.068 & $\begin{array}{l}1.015 \\
1.123\end{array}$ & 0.011 & 1.186 & $\begin{array}{l}1.109 \\
1.267\end{array}$ & $<0.001$ & 1.165 & $\begin{array}{l}1.102 \\
1.233\end{array}$ & $<0.001$ & 1.000 & $\begin{array}{l}0.965 \\
1.036\end{array}$ & 0.993 & 1.037 & $\begin{array}{l}0.988 \\
1.088 \\
\end{array}$ & 0.136 \\
\hline \multicolumn{16}{|l|}{ Region } \\
\hline \multicolumn{16}{|l|}{ Eastern (reference) } \\
\hline Central & 1.213 & $\begin{array}{l}1.149 \\
1.280\end{array}$ & $<0.001$ & 1.205 & $\begin{array}{l}1.122 \\
1.294\end{array}$ & $<0.001$ & 1.178 & $\begin{array}{l}1.109 \\
1.252\end{array}$ & $<0.001$ & 1.320 & $\begin{array}{l}1.271 \\
1.371\end{array}$ & $<0.001$ & 1.375 & $\begin{array}{l}1.302 \\
1.453\end{array}$ & $<0.001$ \\
\hline Western & 1.444 & $\begin{array}{l}1.369 \\
1.522\end{array}$ & $<0.001$ & 1.423 & $\begin{array}{l}1.326 \\
1.526\end{array}$ & $<0.001$ & 1.503 & $\begin{array}{l}1.417 \\
1.594\end{array}$ & $<0.001$ & 1.492 & $\begin{array}{l}1.437 \\
1.549 \\
\end{array}$ & $<0.001$ & 1.670 & $\begin{array}{l}1.584 \\
1.761\end{array}$ & $<0.001$ \\
\hline \multicolumn{16}{|c|}{ Educational attainment } \\
\hline \multicolumn{16}{|c|}{ Illiterate (reference) } \\
\hline Primary school & 0.827 & $\begin{array}{l}0.780 \\
0.877\end{array}$ & $<0.001$ & 0.750 & $\begin{array}{l}0.695 \\
0.809\end{array}$ & $<0.001$ & 0.721 & $\begin{array}{l}0.677 \\
0.769 \\
\end{array}$ & $<0.001$ & 0.859 & $\begin{array}{l}0.822 \\
0.897 \\
\end{array}$ & $<0.001$ & 0.775 & $\begin{array}{l}0.730 \\
0.822\end{array}$ & $<0.001$ \\
\hline $\begin{array}{l}\text { Junior middle } \\
\text { school }\end{array}$ & 0.716 & $\begin{array}{l}0.667 \\
0.768 \\
\end{array}$ & $<0.001$ & 0.655 & $\begin{array}{l}0.597 \\
0.719 \\
\end{array}$ & $<0.001$ & 0.625 & $\begin{array}{l}0.578 \\
0.676 \\
\end{array}$ & $<0.001$ & 0.700 & $\begin{array}{l}0.665 \\
0.736 \\
\end{array}$ & $<0.001$ & 0.704 & $\begin{array}{l}0.657 \\
0.754 \\
\end{array}$ & $<0.001$ \\
\hline $\begin{array}{l}\text { Senior middle } \\
\text { school }\end{array}$ & 0.592 & $\begin{array}{l}0.540 \\
0.649\end{array}$ & $<0.001$ & 0.540 & $\begin{array}{l}0.477 \\
0.611\end{array}$ & $<0.001$ & 0.522 & $\begin{array}{l}0.471 \\
0.578\end{array}$ & $<0.001$ & 0.652 & $\begin{array}{l}0.612 \\
0.694\end{array}$ & $<0.001$ & 0.669 & $\begin{array}{l}0.612 \\
0.730\end{array}$ & $<0.001$ \\
\hline $\begin{array}{l}\text { University/college } \\
\text { or above }\end{array}$ & 0.493 & $\begin{array}{l}0.429 \\
0.565 \\
\end{array}$ & $<0.001$ & 0.474 & $\begin{array}{l}0.393 \\
0.572 \\
\end{array}$ & $<0.001$ & 0.438 & $\begin{array}{l}0.375 \\
0.512 \\
\end{array}$ & $<0.001$ & 0.602 & $\begin{array}{l}0.551 \\
0.657 \\
\end{array}$ & $<0.001$ & 0.740 & $\begin{array}{l}0.657 \\
0.833 \\
\end{array}$ & $<0.001$ \\
\hline \multicolumn{16}{|c|}{ Local ranking of average household income } \\
\hline \multicolumn{16}{|c|}{ Lowest $<$ percentile 20 (reference) } \\
\hline $\begin{array}{l}\text { Low percentile } \\
20-39\end{array}$ & 0.803 & $\begin{array}{l}0.753 \\
0.855 \\
\end{array}$ & $<0.001$ & 0.819 & $\begin{array}{l}0.754 \\
0.891 \\
\end{array}$ & $<0.001$ & 0.789 & $\begin{array}{l}0.735 \\
0.846 \\
\end{array}$ & $<0.001$ & 0.802 & $\begin{array}{l}0.766 \\
0.840 \\
\end{array}$ & $<0.001$ & 0.791 & $\begin{array}{l}0.744 \\
0.842 \\
\end{array}$ & $<0.001$ \\
\hline
\end{tabular}


Table 3. Continued

\begin{tabular}{|c|c|c|c|c|c|c|c|c|c|c|c|c|c|c|c|}
\hline \multirow[b]{2}{*}{ VARIABLE } & \multicolumn{3}{|c|}{ MOBILITY } & \multicolumn{3}{|c|}{ SELF-CARE } & \multicolumn{3}{|c|}{ USUAL ACTIVITIES } & \multicolumn{3}{|c|}{ PAIN/DISCOMFORT } & \multicolumn{3}{|c|}{ ANXIETY/DEPRESSION } \\
\hline & $\mathrm{AOR}^{*}$ & $\begin{array}{c}95 \% \\
\text { CI }\end{array}$ & $P$ & AOR & $\begin{array}{c}95 \% \\
\mathrm{CI}\end{array}$ & $\mathrm{P}$ & AOR & $\begin{array}{c}(95 \% \\
\text { CI }\end{array}$ & $\mathbf{P}$ & AOR & $\begin{array}{l}(95 \% \\
\mathrm{CI})\end{array}$ & $P$ & AOR & $\begin{array}{l}(95 \% \\
\mathrm{CI})\end{array}$ & $P$ \\
\hline $\begin{array}{l}\text { Middle percentile } \\
40-59\end{array}$ & 0.703 & $\begin{array}{l}0.658 \\
0.752\end{array}$ & $<0.001$ & 0.732 & $\begin{array}{l}0.671 \\
0.799 \\
\end{array}$ & $<0.001$ & 0.690 & $\begin{array}{l}0.641 \\
0.743 \\
\end{array}$ & $<0.001$ & 0.758 & $\begin{array}{c}0.723 \\
0.794 \\
\end{array}$ & $<0.001$ & 0.684 & $\begin{array}{l}0.641 \\
0.730\end{array}$ & $<0.001$ \\
\hline $\begin{array}{l}\text { High percentile } \\
60-79\end{array}$ & 0.651 & $\begin{array}{l}0.608 \\
0.696 \\
\end{array}$ & $<0.001$ & 0.669 & $\begin{array}{l}0.612 \\
0.732 \\
\end{array}$ & $<0.001$ & 0.637 & $\begin{array}{l}0.591 \\
0.686 \\
\end{array}$ & $<0.001$ & 0.679 & $\begin{array}{l}0.647 \\
0.712 \\
\end{array}$ & $<0.001$ & 0.623 & $\begin{array}{l}0.584 \\
0.666\end{array}$ & $<0.001$ \\
\hline $\begin{array}{l}\text { Highest } \geq \\
\text { percentile } 80\end{array}$ & 0.627 & $\begin{array}{l}0.585 \\
0.671\end{array}$ & $<0.001$ & 0.654 & $\begin{array}{l}0.596 \\
0.717\end{array}$ & $<0.001$ & 0.622 & $\begin{array}{l}0.576 \\
0.671\end{array}$ & $<0.001$ & 0.647 & $\begin{array}{l}0.616 \\
0.679\end{array}$ & $<0.001$ & 0.580 & $\begin{array}{l}0.541 \\
0.621\end{array}$ & $<0.001$ \\
\hline
\end{tabular}

\section{Employment}

Employed (reference)

\begin{tabular}{|c|c|c|c|c|c|c|c|c|c|c|c|c|c|c|c|}
\hline Retired & 1.884 & $\begin{array}{l}1.748 \\
2.031\end{array}$ & $<0.001$ & 2.522 & $\begin{array}{l}2.278 \\
2.793\end{array}$ & $<0.001$ & 2.132 & $\begin{array}{l}1.959 \\
2.321\end{array}$ & $<0.001$ & 1.072 & $\begin{array}{l}1.017 \\
1.130\end{array}$ & 0.010 & 0.981 & $\begin{array}{c}0.907 \\
1.061\end{array}$ & 0.634 \\
\hline Student & 0.806 & $\begin{array}{c}0.457 \\
1.421\end{array}$ & 0.456 & 0.884 & $\begin{array}{l}0.458 \\
1.705\end{array}$ & 0.713 & 0.591 & $\begin{array}{l}0.313 \\
1.116\end{array}$ & 0.105 & 0.639 & $\begin{array}{l}0.450 \\
0.907\end{array}$ & 0.012 & 0.706 & $\begin{array}{l}0.479 \\
1.042\end{array}$ & $<0.001$ \\
\hline Unemployed & 2.670 & $\begin{array}{l}2.521 \\
2.826\end{array}$ & $<0.001$ & 3.345 & $\begin{array}{l}3.096 \\
3.614\end{array}$ & $<0.001$ & 3.097 & $\begin{array}{l}2.907 \\
3.299\end{array}$ & $<0.001$ & 1.571 & $\begin{array}{l}1.508 \\
1.637\end{array}$ & $<0.001$ & 1.675 & $\begin{array}{l}1.584 \\
1.771\end{array}$ & $<0.001$ \\
\hline
\end{tabular}

\section{Marital status:}

Never married/Single (reference)

\begin{tabular}{lccccccccccccccccc}
\hline \multirow{2}{*}{ Married } & 0.489 & $\begin{array}{c}0.430, \\
0.556\end{array}$ & $<0.001$ & 0.426 & $\begin{array}{c}0.365, \\
0.497\end{array}$ & $<0.001$ & 0.417 & $\begin{array}{c}0.365, \\
0.476\end{array}$ & $<0.001$ & 0.798 & $\begin{array}{c}0.725, \\
0.879\end{array}$ & $<0.001$ & 0.546 & $\begin{array}{c}0.487, \\
0.612\end{array}$ & $<0.001$ \\
\hline \multirow{2}{*}{ Divorced } & \multirow{2}{*}{0.608} & $\begin{array}{c}0.528, \\
0.699\end{array}$ & $<0.001$ & \multirow{2}{*}{0.547} & $\begin{array}{c}0.462, \\
0.648\end{array}$ & $<0.001$ & 0.516 & $\begin{array}{c}0.446, \\
0.597\end{array}$ & $<0.001$ & 0.927 & $\begin{array}{c}0.833, \\
1.033\end{array}$ & 0.170 & 0.676 & $\begin{array}{c}0.593, \\
0.772\end{array}$ & $<0.001$ \\
\hline \multirow{2}{*}{ Widowed } & \multirow{2}{*}{0.814} & $\begin{array}{r}0.660 \\
1.005\end{array}$ & \multirow{2}{*}{0.055} & \multirow{2}{*}{0.744} & $\begin{array}{c}0.570, \\
0.971\end{array}$ & $<0.001$ & 0.729 & $\begin{array}{c}0.582, \\
0.914\end{array}$ & 0.006 & 1.158 & $\begin{array}{c}0.997, \\
1.345\end{array}$ & 0.055 & 1.088 & $\begin{array}{c}0.911, \\
1.300\end{array}$ & 0.350 \\
\hline
\end{tabular}

Two-week morbidity

Yes (reference)

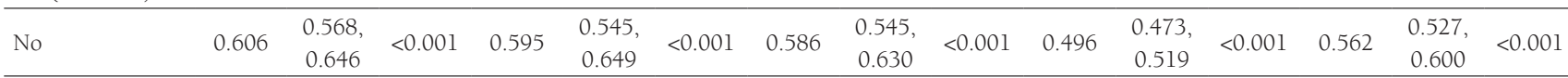

\section{Chronic disease}

Yes (reference)

\begin{tabular}{lllllllllllllllll}
\hline \multirow{2}{*}{ No } & 0.489 & $\begin{array}{c}0.458, \\
0.523\end{array}$ & $<0.001$ & 0.552 & $\begin{array}{c}0.503, \\
0.605\end{array}$ & $<0.001$ & 0.488 & $\begin{array}{c}0.452, \\
0.526\end{array}$ & $<0.001$ & 0.488 & $\begin{array}{c}0.465, \\
0.512\end{array}$ & $<0.001$ & 0.503 & 0.47, \\
\end{tabular}

\section{One-year hospital admission}

Yes (reference)

\begin{tabular}{|c|c|c|c|c|c|c|c|c|c|c|c|c|c|c|c|}
\hline No & 0.468 & $\begin{array}{l}0.443 \\
0.494 \\
\end{array}$ & $<0.001$ & 0.459 & $\begin{array}{l}0.428 \\
0.492 \\
\end{array}$ & $<0.001$ & 0.440 & $\begin{array}{l}0.414 \\
0.467 \\
\end{array}$ & $<0.001$ & 0.520 & $\begin{array}{l}0.499 \\
0.543\end{array}$ & $<0.001$ & 0.525 & $\begin{array}{l}0.497 \\
0.555 \\
\end{array}$ & $<0.001$ \\
\hline \multicolumn{16}{|c|}{ Smoking } \\
\hline \multicolumn{16}{|c|}{ Yes (reference) } \\
\hline No & 1.188 & $\begin{array}{l}1.118 \\
1.263 \\
\end{array}$ & $<0.001$ & 1.323 & $\begin{array}{l}1.219 \\
1.437\end{array}$ & $<0.001$ & 1.279 & $\begin{array}{l}1.194, \\
1.370\end{array}$ & $<0.001$ & 1.021 & $\begin{array}{c}0.977 \\
1.066\end{array}$ & 0.360 & 1.055 & $\begin{array}{l}0.992, \\
1.122 \\
\end{array}$ & $<0.001$ \\
\hline \multicolumn{16}{|c|}{ Drinking } \\
\hline \multicolumn{16}{|c|}{ Yes (reference) } \\
\hline No & 1.338 & $\begin{array}{l}1.254 \\
1.427 \\
\end{array}$ & $<0.001$ & 1.885 & $\begin{array}{l}1.716 \\
2.069 \\
\end{array}$ & $<0.001$ & 1.649 & $\begin{array}{l}1.529 \\
1.778\end{array}$ & $<0.001$ & 0.987 & $\begin{array}{c}0.945 \\
1.031\end{array}$ & 0.565 & 1.088 & $\begin{array}{l}1.022 \\
1.158 \\
\end{array}$ & $<0.001$ \\
\hline \multicolumn{16}{|c|}{ Physical exercise } \\
\hline \multicolumn{16}{|c|}{ Yes (reference) } \\
\hline No & 2.091 & $\begin{array}{l}1.970 \\
2.219\end{array}$ & $<0.001$ & 2.549 & $\begin{array}{l}2.348 \\
2.767\end{array}$ & $<0.001$ & 2.325 & $\begin{array}{l}2.172 \\
2.489\end{array}$ & $<0.001$ & 1.299 & $\begin{array}{l}1.249 \\
1.352\end{array}$ & $<0.001$ & 1.424 & $\begin{array}{l}1.345 \\
1.508\end{array}$ & $<0.001$ \\
\hline
\end{tabular}

AOR - adjusted odds ratio, $\mathrm{CI}-95 \%$ confidence interval

states of "11121" (5.87\%), "11122" (1.64\%), "11112" (1.24\%), "21121" (0.91\%), "22222" (0.76\%), "22221" $(0.73 \%)$, and "21111" (0.68\%). This generated a very high mean score of the utility index: $0.985(S D=0.056)$. Details about the distributional information of the 161 EQ-5D-3L health states, and the median and IQR of utility index can be found in the supplementary file (Table S1 and S2 in the Online Supplementary Document).

Similarly, the distribution of the VAS score was also negatively skewed (Figure 3). More than $40 \%$ of respondents reported a higher than 90 VAS score. But the mean and median values of VAS were similar: 80.91 $(S D=13.74)$ vs 80.00 (IQR: 70.00 - 90.00). Details about the median and IQR of VAS score can be found in the supplementary file (Table S2 in the Online Supplementary Document). The VAS scores were moderately 


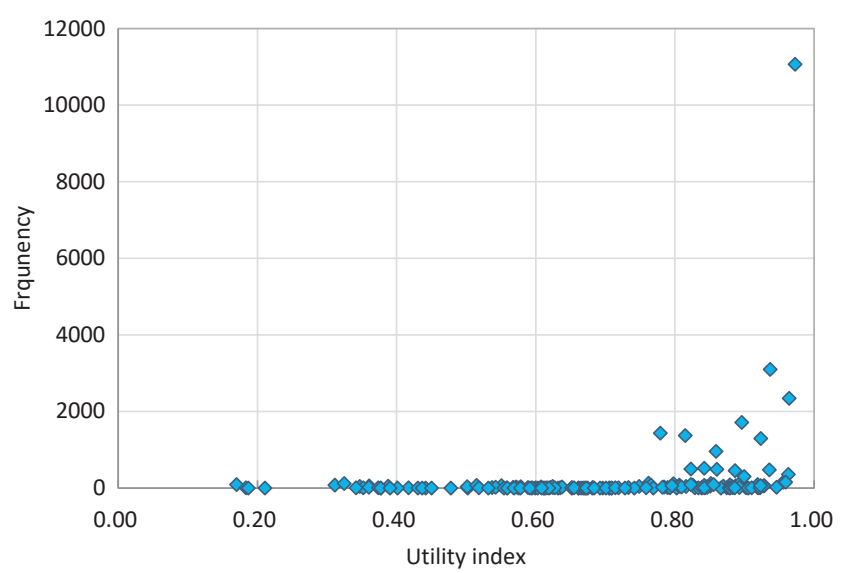

Figure 2. Distribution of EQ-5D-3L utility index scores in the respondents with a health problem.

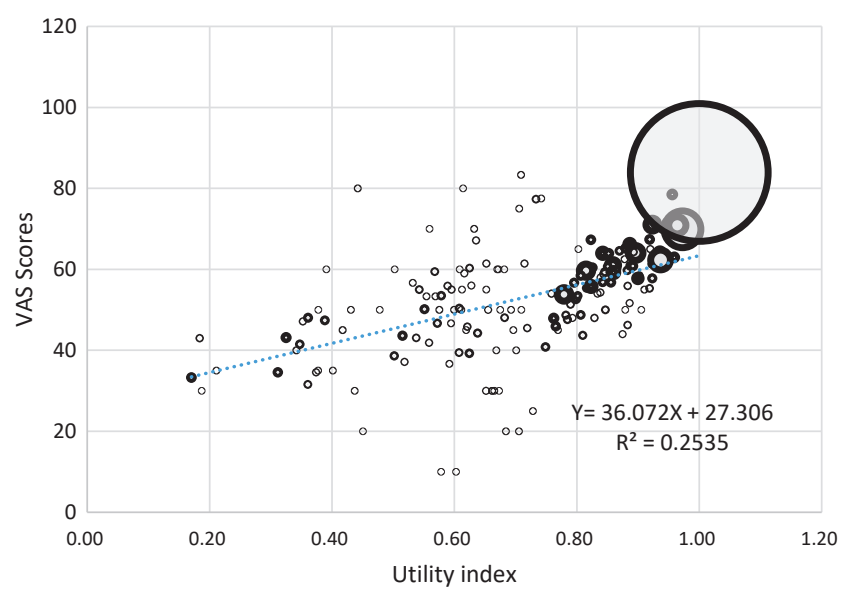

Figure 4. Correlation between EQ-5D-3L VAS scores and utility index $(r=0.4537, P<0.05)$. Note: The size of the bubbles represents the frequency of individuals with a corresponding combination of the two scores

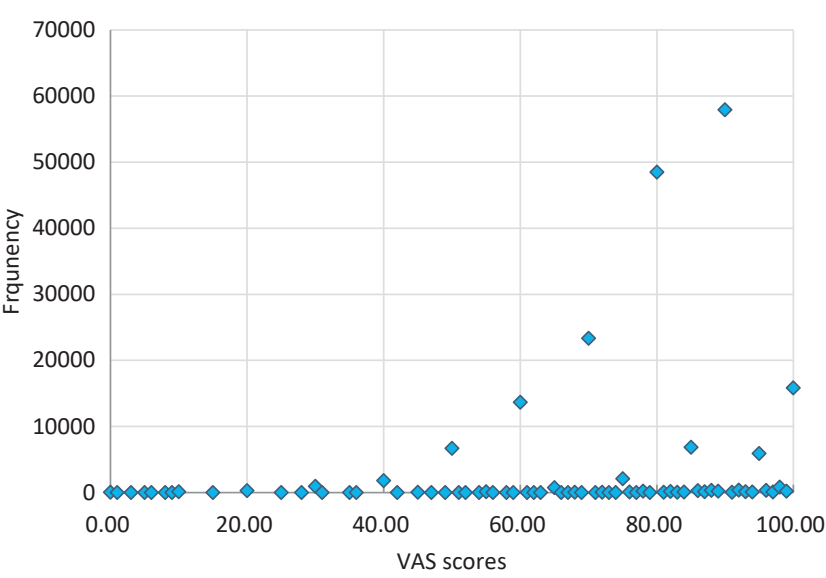

Figure 3. Distribution of EQ-5D-3L VAS scores in the respondents. (Mean $=80.91, \mathrm{SD}=13.74$, Median $=80.00, \mathrm{IQR}=70.00-90.00)$.

correlated with the utility index scores $(r=0.4537, P<0.05)$ in the total sample (Figure 4), as well as in the subsamples stratified by gender and age (Figure S1 to S9 in the Online Supplementary Document).

Men and rural residents had higher VAS scores (but not in the utility index) than women and urban residents. Younger respondents had higher VAS and utility index scores than their older counterparts. Socioeconomic gradients were evident, and those with a higher socio-economic status (eg, better educated, higher income, employed and living in developed areas) had higher utility index and VAS scores. Those who did not smoke or drink had a lower utility index, but not in VAS scores (Table 1 and Table 4). In addition, the effect size of differences in age, region, education attainment, income level, employment status, marital status, two-week morbidity, chronic disease, hospitalisation, and exercising on the utility index exceeded the medium effect size of 0.5 (Table 4). The median and IQR results also supported these findings (Table S2 in the Online Supplementary Document).

Table 4. Factors associated with VAS and utility index scores - results of multiple linear and Tobit regression analyses

\begin{tabular}{|c|c|c|c|c|c|c|c|c|c|c|}
\hline \multirow[b]{2}{*}{ VARIABLE } & \multicolumn{5}{|c|}{ EQ-5D VAS } & \multicolumn{5}{|c|}{ EQ-5D UTILITY } \\
\hline & $\beta^{*}$ & SE & $95 \%$ CI & $\mathbf{P}$ & $\begin{array}{l}\text { Effect } \\
\text { size }\end{array}$ & $\beta$ & SE & $95 \%$ CI & $P$ value & $\begin{array}{c}\text { Effect } \\
\text { size }\end{array}$ \\
\hline \multicolumn{11}{|c|}{ Gender } \\
\hline \multicolumn{11}{|c|}{ Male (reference) } \\
\hline Female & -0.163 & 0.072 & $-0.304,-0.023$ & 0.023 & -0.01 & 0.003 & 0.002 & $-0.001,0.006$ & 0.114 & 0.05 \\
\hline \multicolumn{11}{|l|}{ Age } \\
\hline \multicolumn{11}{|c|}{ 15-24 (reference) } \\
\hline $25-34$ & -2.406 & 0.120 & $-2.642,-2.171$ & $<0.001$ & -0.18 & -0.051 & 0.006 & $-0.063,-0.039$ & $<0.001$ & -0.91 \\
\hline $35-44$ & -5.171 & 0.128 & $-5.422,-4.920$ & $<0.001$ & -0.38 & -0.105 & 0.006 & $-0.116,-0.093$ & $<0.001$ & -1.87 \\
\hline $45-54$ & -7.548 & 0.131 & $-7.804,-7.292$ & $<0.001$ & -0.55 & -0.136 & 0.006 & $-0.147,-0.124$ & $<0.001$ & -2.42 \\
\hline $55-64$ & -9.507 & 0.140 & $-9.782,-9.233$ & $<0.001$ & -0.69 & -0.156 & 0.006 & $-0.168,-0.145$ & $<0.001$ & -2.79 \\
\hline $65-74$ & -11.709 & 0.165 & $-12.033,-11.385$ & $<0.001$ & -0.85 & -0.180 & 0.006 & $-0.192,-0.168$ & $<0.001$ & -3.21 \\
\hline $75+$ & -13.949 & 0.208 & $-14.355,-13.542$ & $<0.001$ & -1.02 & -0.236 & 0.006 & $-0.248,-0.223$ & $<0.001$ & -4.20 \\
\hline \multicolumn{11}{|l|}{ Location } \\
\hline \multicolumn{11}{|c|}{ Urban (reference) } \\
\hline Rural & 0.731 & 0.062 & $0.609,0.854$ & $<0.001$ & 0.05 & -0.002 & 0.001 & $-0.005,0.001)$ & 0.195 & -0.03 \\
\hline
\end{tabular}


Table 4. Continued

\begin{tabular}{|c|c|c|c|c|c|c|c|c|c|c|}
\hline \multirow[b]{2}{*}{ VARIABLE } & \multicolumn{5}{|c|}{ EQ-5D VAS } & \multicolumn{5}{|c|}{ EQ-5D UTILITY } \\
\hline & $\beta^{*}$ & SE & $95 \%$ CI & $\mathbf{P}$ & $\begin{array}{l}\text { Effect } \\
\text { size }\end{array}$ & $\beta$ & SE & $95 \%$ CI & $P$ value & $\begin{array}{c}\text { Effect } \\
\text { size }\end{array}$ \\
\hline \multicolumn{11}{|l|}{ Region } \\
\hline \multicolumn{11}{|l|}{ Eastern (reference) } \\
\hline Central & -1.439 & 0.067 & $-1.570,-1.309$ & $<0.001$ & -0.10 & -0.019 & 0.002 & $-0.022,-0.016$ & $<0.001$ & -0.34 \\
\hline Western & -2.715 & 0.065 & $-2.843,-2.588$ & $<0.001$ & -0.20 & -0.031 & 0.002 & $-0.034,-0.028$ & $<0.001$ & -0.55 \\
\hline \multicolumn{11}{|l|}{ Educational attainment } \\
\hline \multicolumn{11}{|l|}{ Illiterate (reference) } \\
\hline Primary school & 1.964 & 0.113 & $1.743,2.186$ & $<0.001$ & 0.14 & 0.020 & 0.002 & $0.016,0.023$ & $<0.001$ & 0.35 \\
\hline Junior middle school & 3.151 & 0.116 & $2.925,3.378$ & $<0.001$ & 0.23 & 0.036 & 0.002 & $0.032,0.04$ & $<0.001$ & 0.65 \\
\hline Senior middle school & 2.996 & 0.129 & $2.744,3.249$ & $<0.001$ & 0.22 & 0.043 & 0.003 & $0.038,0.048$ & $<0.001$ & 0.76 \\
\hline University/college or above & 2.754 & 0.142 & $2.475,3.033$ & $<0.001$ & 0.20 & 0.041 & 0.003 & $0.035,0.048$ & $<0.001$ & 0.74 \\
\hline \multicolumn{11}{|c|}{ Local ranking of average household income } \\
\hline \multicolumn{11}{|c|}{ Lowest (<percentile 20) (reference) } \\
\hline Low (percentile 20-39) & 1.371 & 0.095 & $1.185,1.556$ & $<0.001$ & 0.10 & 0.021 & 0.002 & $0.017,0.024$ & $<0.001$ & 0.37 \\
\hline Middle (percentile 40-59) & 2.042 & 0.092 & $1.861,2.223$ & $<0.001$ & 0.15 & 0.031 & 0.002 & $0.027,0.034$ & $<0.001$ & 0.54 \\
\hline High (percentile 60-79) & 2.376 & 0.091 & $2.196,2.555$ & $<0.001$ & 0.17 & 0.038 & 0.002 & $0.034,0.041$ & $<0.001$ & 0.67 \\
\hline Highest ( $\geq$ percentile 80$)$ & 2.841 & 0.091 & $2.662,3.021$ & $<0.001$ & 0.21 & 0.041 & 0.002 & $0.037,0.044$ & $<0.001$ & 0.72 \\
\hline \multicolumn{11}{|l|}{ Employment } \\
\hline \multicolumn{11}{|l|}{ Employed (reference) } \\
\hline Retired & -0.846 & 0.112 & $-1.066,-0.627$ & $<0.001$ & -0.06 & -0.022 & 0.002 & $-0.027,-0.018$ & $<0.001$ & -0.40 \\
\hline Student & 0.430 & 0.151 & $0.134,0.727$ & 0.004 & 0.03 & 0.017 & 0.009 & $-0.002,0.035$ & $<0.001$ & 0.29 \\
\hline Unemployed & -2.942 & 0.097 & $-3.132,-2.753$ & $<0.001$ & -0.21 & -0.063 & 0.002 & $-0.066,-0.059$ & $<0.001$ & -1.11 \\
\hline \multicolumn{11}{|c|}{ Marital status } \\
\hline \multicolumn{11}{|c|}{ Never married/Single (reference) } \\
\hline Married & 0.687 & 0.120 & $0.452,0.923$ & $<0.001$ & 0.05 & 0.042 & 0.004 & $0.035,0.050$ & $<0.001$ & 0.75 \\
\hline Divorced & -0.315 & 0.184 & $-0.676,0.046$ & 0.087 & -0.02 & 0.023 & 0.004 & $0.015,0.032$ & $<0.001$ & 0.42 \\
\hline Widowed & -1.367 & 0.267 & $-1.89,-0.843$ & $<0.001$ & -0.10 & -0.001 & 0.006 & $-0.013,0.01$ & 0.849 & -0.02 \\
\hline \multicolumn{11}{|l|}{ Two-week morbidity } \\
\hline \multicolumn{11}{|l|}{ Yes (reference) } \\
\hline No & 3.282 & 0.105 & $3.076,3.487$ & $<0.001$ & 0.24 & 0.059 & 0.002 & $0.055,0.063$ & $<0.001$ & 1.05 \\
\hline \multicolumn{11}{|l|}{ Chronic disease } \\
\hline \multicolumn{11}{|l|}{ Yes (reference) } \\
\hline No & 4.756 & 0.103 & $4.554,4.958$ & $<0.001$ & 0.35 & 0.063 & 0.002 & $0.059,0.066$ & $<0.001$ & 1.12 \\
\hline \multicolumn{11}{|c|}{ One-year hospital admission } \\
\hline \multicolumn{11}{|c|}{ Yes (reference) } \\
\hline No & 4.049 & 0.119 & $3.816,4.282$ & 0.281 & 0.29 & 0.066 & 0.002 & $0.062,0.070$ & $<0.001$ & 1.18 \\
\hline \multicolumn{11}{|l|}{ Smoking } \\
\hline \multicolumn{11}{|l|}{ Yes (reference) } \\
\hline No & -0.083 & 0.077 & $-0.234,0.068$ & $<0.001$ & -0.01 & -0.009 & 0.002 & $-0.012,-0.006$ & $<0.001$ & -0.16 \\
\hline \multicolumn{11}{|l|}{ Drinking } \\
\hline Yes (reference) & & & & & & & & & & \\
\hline No & -0.799 & 0.074 & $-0.944,-0.654$ & $<0.001$ & -0.06 & -0.007 & 0.002 & $-0.011,-0.004$ & $<0.001$ & -0.13 \\
\hline
\end{tabular}

\section{Physical exercise}

Yes (reference)

\begin{tabular}{lllllllllllll}
\hline No & -1.190 & 0.069 & $-1.324,-1.055$ & $<0.001$ & -0.09 & -0.037 & 0.002 & $-0.040,-0.034$ & $<0.001$ & -0.66
\end{tabular}

$\beta$ - standardised beta coefficient in the regression models, SE - standard error, 95\% CI - 95\% confidence interval

In the supplementary file (Table S3 and Table S5 in the Online Supplementary Document), we presented the means and standard deviations of the VAS and utility index scores, respectively, by gender, age, region and residency. The respondents living in the eastern developed region had the highest VAS and utility index scores in all age groups. In contrast, those living in the western under-developed region had the lowest VAS and utility index scores. The urban-rural disparities appeared to vary by regions. Urban residents residing in the central region had a higher utility index score for all age groups than their rural counterparts. But in the eastern and western regions, urban residents aged between 15 and 54 years had a lower utility index than their rural counterparts (Table S5 in the Online Supplementary Document). Similar results can be found in the medians and IQRs of the VAS and utility index scores presented in the supplementary file (Table S4 and Table S6 in the Online Supplementary Document). 


\section{DISCUSSION}

To the best of our knowledge, this is the first paper to provide Chinese EQ-5D-3L population norms based on a large sample using the nationally representative preference-based value sets. The population norms, presented in the percentage of reported problems, means (standard deviations), and medians (interquartile ranges) of VAS and utility index scores, can serve as reference for comparative purposes in HRQoL studies and health economic evaluation studies in China.

Overall, the Chinese people have relatively higher utility index scores compared with those from other countries $[4,7,11,16,18,19,24,27]$, although the mean value $(0.985)$ is a bit closer to those in Singapore $(0.950)$ [25] and Korea (0.958) [54]. The European value sets would bring the mean utility index score down to 0.951, which is still high compared with other populations [27,55]. Previous studies using the value sets derived from a small sample of urban populations in big cities may have also underestimated the HRQoL of the Chinese populations (Table S7 in the Online Supplementary Document).

Ceiling effects are profound in the Chinese populations (especially in the young groups), with $84.2 \%$ reporting no health problems compared with $41.3 \%$ in Portugal [19], 47.1\% in Poland, 62.4\% in Spain [47], $68.0 \%$ in Japan [4], and 79.0\% in Singapore [25]. Similar findings were also reported in previous studies in China $[31,39,44]$. Overall, ceiling effects of the EQ-5D-3L instrument were relatively high in Asian countries $[4,25,27,31,39,44]$. However, the percentage of reported problems in China is not always the lowest in comparison with other countries. Some American/Western European countries (such as Denmark, Germany, Sweden and Switzerland) reported even lower levels of problems in some dimensions (Table S7 in the Online Supplementary Document). The differences can be attributable to multiple factors in relation to values, culture, and traditions (eg, tolerance to various health problems) [56,57]. As a result, the use and interpretation of the EQ-5D-3L utility index needs to be cautious. Empirical evidence shows that Asian populations are less likely to report health problems than their European counterparts, inflating the utility index [27]. It is important to note that the percentage of respondents in this study reporting problems on the five dimensions of the EQ-5D-3L are consistently lower compared with the populations in other countries $[3,4,7,11,13,16$ $19,22,24,25,27,47,54,58,59]$. Similar to studies undertaken in other countries, pain/discomfort was the most frequently reported problem $[11,60]$. But only about $12 \%$ of the Chinese respondents reported problems in pain/discomfort, much less than those from other countries, which could be as high as $65.0 \%[3,4,7,11,13,16$ $19,22,24,25,27,47,54,58,59]$. The next frequently reported problems in the Chinese populations were mobility (5.9\%) and anxiety/depression (5.3\%), again at a level lower than other countries $[3,4,7,11,13,16,18,19$, $22,24,27,47,54,58,59]$. The only exceptions are the lower level of reported problems in mobility $(3.6 \%)$ in the Singapore population [25] and the lower level of reported problems in anxiety/depression in the Netherlands (3.5\%) and German (4.3\%) populations [11,27].

Unlike the utility index, the average level (80.91) of VAS scores in this study population is similar to those in New Zealand (80.8) [59], Sri Lanka (81.0) [7], Switzerland (81.7) [18] and a previous study in China (80.4) [27], lower than those in Denmark (83.7) [22], Sweden (83.3) [58], the UK (82.8) [13] and the Netherlands (82.0) [17], but higher than those in some other countries (ranging from 71.1 to 80.0) $[3,11,19,24]$. Further analyses demonstrated a wide distribution of VAS scores in those who reported no health problems, indicating a low discriminatory power of the utility index (Table S7 in the Online Supplementary Document).

There exists a gender gap in HRQoL of the Chinese populations. Overall, male respondents were less likely to report health problems than female respondents, resulting in higher VAS and utility index scores. However, after controlling for variations in other factors, the effect size in the differences in gender on the utility index was only 0.05. This phenomenon was also observed in studies in Singapore [25] and other studies in China[31,35,39,61]. Szende and colleagues believe that gender plays a small role in explaining HRQoL [11]. It is worth noting that gender variations in utility index and VAS scores were not always consistent. Women had lower scores in VAS than men, but not in utility index. These inconsistencies may be due to the conceptual differences in the two measurements [62]. VAS reflects a direct individual real-time rating considering all aspects of health; whereas, utility index is an indirect measurement, using past time value sets to estimate current states considering limited dimensions of health [38]. Women may have a relatively higher expectation on health, resulting in lower ratings on VAS $[31,44]$. Similar to other studies $[4,6,7,11,13,16,19,22,24$, $31,39,60,63]$, we found that older age is associated with lower HRQoL (with an effect size of 0.91-4.20 on the utility index). China is currently experiencing unprecedented rapid transition to an ageing society as a result of the decades long family planning policy [64], which could lead to an overall decline in HRQoL of the entire population. 
Regional and residential disparities in HRQoL in the Chinese populations are evident. Those who resided in the eastern developed region had higher HRQoL than their central and western counterparts, with a medium effect size (0.34-0.55) on the utility index. Rural respondents in this study had higher VAS ratings than their urban counterparts although they were more likely to report problems in mobility, self-care, and usual activities. Unlike the utility index measurement applying past time value sets, VAS scores reflect real time individual subjective ratings. Rural residents may have relatively lower expectation on health, resulting in higher ratings on VAS [31,44]. According to the Guide Book of EQ-5D-3L, both utility index and VAS scores should be presented in result reporting. Young rural residents ( $\leq 55$ years) had higher utility index scores than their urban counterparts, especially in the eastern and western regions. By contrast, old rural residents ( $\geq 65$ years) had lower utility index scores than their urban counterparts. These results are consistent with findings reported in previous studies in China and elsewhere $[31,35,39,44,61,63]$. However, the urban-rural differences revealed in this study is small based on effect size (0.03-0.05). Nevertheless, it is important to note that population mobility is high in China: young people in rural and undeveloped regions are increasingly moving to urban and more developed regions $[64,65]$. This could exacerbate the aging process in rural areas, lowering the utility index of rural residents.

Socioeconomic gradients in HRQoL as measured by the three indicators (percentage of reported problems, utility index and VAS scores) deserve increased attention. We found that those with a higher socio-economic status (eg, richer, better educated, employed and married) have significantly higher HRQoL than others, after controlling for variations in demographic and health characteristics. These results are consistent with findings from previous studies in China and elsewhere $[4,6,7,11,13,16,22,25,31,39,60,63]$. The effect size on the utility index reached $0.35-0.76$ for education, $0.37-0.74$ for income, 0.29-1.11 for employment, and 0.02-0.75 for marital status.

This study adds additional evidence to support the proposed association between physical activity and HRQoL $[4,6,7,11,13,16,22,31,39,60,63]$. The effect size of regular exercise $(0.66)$ on the utility index shows clinical significance. However, the associations between HRQoL and smoking and drinking are small, albeit statistically significant, failing to reach a clinical meaningful level according to the effect size $(0.16$ for smoking and 0.13 for drinking). Positive associations between HRQoL and smoking and drinking were reported in previous studies conducted elsewhere in China [66-68].

Limitations: There are several limitations in this study. First, this is a cross-sectional study and no causal relationships should be assumed for the findings. Second, there is a high ceiling effect for the EQ-5D-3L utility index. Although the EQ-5D-5L descriptive system may improve the discriminatory power, its ceiling effect is likely to stay high in the Chinese populations $[69,70]$. Further studies should examine the cultural responsiveness of the EQ-5D-3L instrument [56] and the appropriateness of the translated wording and phrasing (language) of its descriptive system [71] in the Chinese context. We reported mean values and standard deviations of the EQ-5D utility index in line with other studies despite its high ceiling effect $[4,15,24,27,47]$. Non-normal distributions of EQ-5D utility index scores are common in all EQ-5D studies: full health status was reported in $79 \%$ Singaporean populations, $68 \%$ in Japanese populations, and more than $50 \%$ of populations in Poland, France and Spain $[4,18,24,27,47,60]$. Third, the EQ-5D instruments measure limited dimensions of health due to a small number (five) of items. The small item number made it easier to generate a utility index score, which is often absent from a more comprehensive instrument (for example the SF-36) [38,72,73]. However, the implications of the utility index need to be interpreted with caution, and in conjunction with analyses of the problems reported and VAS scores.

\section{CONCLUSIONS}

This paper provides population norms for the EQ-5D-3L in China stratified by age, gender and region based on the 2018 population preference-based value sets derived from a national representative sample. The norms can be used as a reference for health economic evaluation studies. Overall, 15.8\% of respondents reported a health problem, with pain/discomfort being the most commonly reported problem. Compared with other populations, the Chinese people have high scores in VAS and utility index. Those who are richer, better educated, employed, married, and live in developed areas have higher scores in both VAS and utility index than others. Further studies are needed to explore the underlying reasons of the sociodemographic differences.

Given the high ceiling effect and low discriminatory power of the utility index, cautions should be taken in presenting and interpreting mean values of the utility index. The meaning and implications of the EQ-5D-3L utility index need to be interpreted in conjunction with other indicators, including the nature and number of 
problems reported, the distributional position of the health state, and the VAS scores. However, we acknowledge that the ceiling effect of the utility index is less serious in some subpopulations, such as the elderly and those with existing illness conditions, as revealed in this study. This suggests that it may be more appropriate to use the EQ-5D-3L instrument in those subpopulations rather than the entire general population. We advocate use of the percentile indicators for presenting population-based results of the EQ-5D-3L utility index and further studies into the health state descriptive system tailored to the specific context of the Chinese populations.

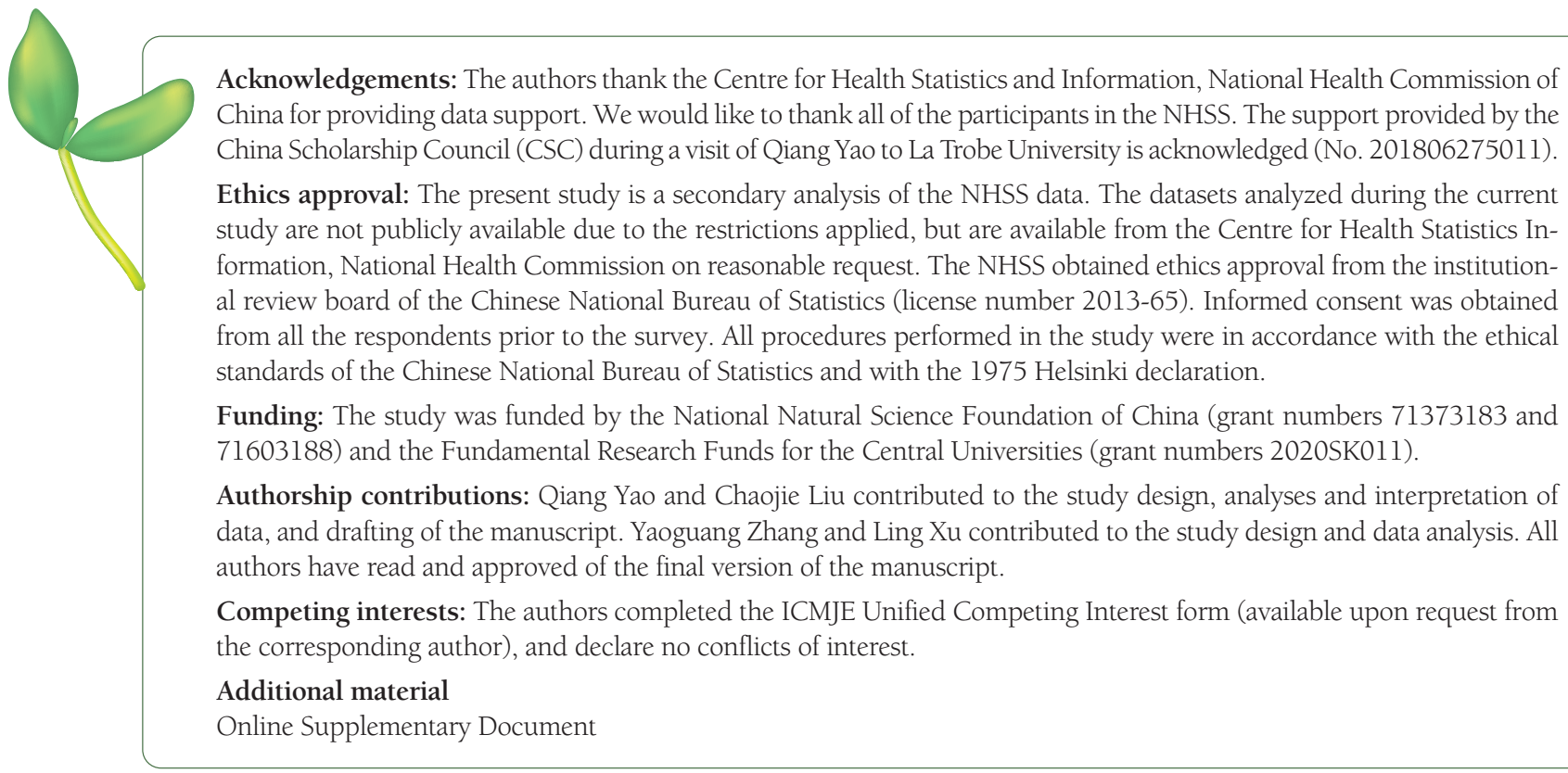

1 Karimi M, Brazier J. Health, health-related quality of life, and quality of life: what is the difference? Pharmacoeconomics. 2016;34:645-9. Medline:26892973 doi:10.1007/s40273-016-0389-9

2 Yin S, Njai R, Barker L, Siegel PZ, Liao Y. Summarizing health-related quality of life (HRQOL): development and testing of a one-factor model. Popul Health Metr. 2016;14:22. Medline:27408606 doi:10.1186/s12963-016-0091-3

3 Scalone L, Cortesi PA. CIAMpICHINI R, Cesana G, Mantovani LG. Health related quality of life norm data of the Italian general population: results using the EQ-5D-3L and EQ-5D-5L instruments. Epidemiol Biostat Public Health. 2015;12:e114571-15.

4 Shiroiwa T, Fukuda T, Ikeda S, Igarashi A, Noto S, Saito S, et al. Japanese population norms for preference-based measures: EQ-5D-3L, EQ-5D-5L, and SF-6D. Qual Life Res. 2016;25:707-19. Medline:26303761 doi:10.1007/s11136-015-1108-2

5 Fryback DG, Dunham NC, Palta M, Hanmer J, Buechner J, Cherepanov D, et al. US norms for six generic health-related quality-of-life indexes from the National Health Measurement study. Med Care. 2007;45:1162. Medline:18007166 doi:10.1097/ MLR.0b013e31814848f1

6 Luo N, Johnson JA, Shaw JW, Feeny D, Coons SJ. Self-reported health status of the general adult US population as assessed by the EQ-5D and Health Utilities Index. Med Care. 2005;43:1078-86. Medline:16224300 doi:10.1097/01.mlr.0000182493.57090.c1

7 Kularatna S, Whitty JA, Johnson NW, Jayasinghe R, Scuffham PA. EQ-5D-3L derived population norms for health related quality of life in Sri Lanka. PLoS One. 2014;9:e108434. Medline:25365171 doi:10.1371/journal.pone.0108434

8 Furlong WJ, Feeny DH, Torrance GW, Barr RD. The Health Utilities Index (HUIß) system for assessing health-related quality of life in clinical studies. Ann Med. 2001;33:375-84. Medline:11491197 doi:10.3109/07853890109002092

9 Fayers PM, Machin D. Quality of Life: The Assessment, Analysis and Interpretation of Patient-Reported Outcomes: Second Edition2013.

10 Devlin NJ, Brooks R. EQ-5D and the EuroQol group: past, present and future. Appl Health Econ Health Policy. 2017;15:12737. Medline:28194657 doi:10.1007/s40258-017-0310-5

11 Szende A, Janssen B, Cabases J. Self-reported population health: an international perspective based on EQ-5D. Dordrecht (The Netherlands): Springer; 2014.

12 Oppe M, Devlin NJ, Szende A. EQ-5D value sets: inventory, comparative review and user guide. Berlin, Germany: Springer; 2007.

13 Kind P, Dolan P, Gudex C, Williams A. Variations in population health status: results from a United Kingdom national questionnaire survey. BMJ. 1998;316:736-41. Medline:9529408 doi:10.1136/bmj.316.7133.736

14 Johnson JA, Pickard AS. Comparison of the EQ-5D and SF-12 health surveys in a general population survey in Alberta, Canada. Med Care. 2000;38:115-21. Medline:10630726 doi:10.1097/00005650-200001000-00013

15 McCaffrey N, Kaambwa B, Currow DC, Ratcliffe J. Health-related quality of life measured using the EQ-5D-5L: South Australian population norms. Health Qual Life Outcomes. 2016;14:133. Medline:27644755 doi:10.1186/s12955-016-0537-0 
16 Clemens S, Begum N, Harper C, Whitty JA, Scuffham PA. A comparison of EQ-5D-3L population norms in Queensland, Australia, estimated using utility value sets from Australia, the UK and USA. Qual Life Res. 2014;23:2375-81. Medline:24676898 doi:10.1007/s11136-014-0676-x

17 König H-H, Bernert S, Angermeyer MC, Matschinger H, Martinez M, Vilagut G, et al. Comparison of population health status in six european countries: results of a representative survey using the EQ-5D questionnaire. Med Care. 2009;47:255-61. Medline:19169128 doi:10.1097/MLR.0b013e318184759e

18 Perneger TV, Combescure C, Courvoisier DS. General Population Reference Values for the French Version of the EuroQol EQ-5D Health Utility Instrument. Value Health. 2010;13:631-5. Medline:20412541 doi:10.1111/j.1524-4733.2010.00727.x

19 Ferreira LN, Ferreira PL, Pereira LN, Oppe M. EQ-5D Portuguese population norms. Qual Life Res. 2014;23:425-30. Medline:23912856 doi:10.1007/s11136-013-0488-4

20 Cunillera O, Tresserras R, Rajmil L, Vilagut G, Brugulat P, Herdman M, et al. Discriminative capacity of the EQ-5D, SF-6D, and SF-12 as measures of health status in population health survey. Qual Life Res. 2010;19:853-64. Medline:20354795 doi:10.1007/s11136-010-9639-z

21 Burström K, Johannesson M, Diderichsen F. Swedish population health-related quality of life results using the EQ-5D. Qual Life Res. 2001;10:621-35. Medline:11822795 doi:10.1023/A:1013171831202

22 Sørensen J, Davidsen M, Gudex C, Pedersen KM, Brønnum-Hansen H. Danish EQ-5D population norms. Scand J Public Health. 2009;37:467-74. Medline:19535407 doi:10.1177/1403494809105286

23 Saarni SI, Härkänen T, Sintonen H, Suvisaari J, Koskinen S, Aromaa A, et al. The impact of 29 chronic conditions on health-related quality of life: a general population survey in Finland using 15D and EQ-5D. Qual Life Res. 2006;15:1403-14. Medline:16960751 doi:10.1007/s11136-006-0020-1

24 Golicki D, Niewada M. General population reference values for 3-level EQ-5D (EQ-5D-3L) questionnaire in Poland. Pol Arch Med Wewn. 2015;125:18-26. Medline:25578383 doi:10.20452/pamw.2638

25 Abdin E, Subramaniam M, Vaingankar JA, Luo N, Chong SA. Population norms for the EQ-5D index scores using Singapore preference weights. Qual Life Res. 2015;24:1545-53. Medline:25394893 doi:10.1007/s11136-014-0859-5

26 Menezes RM, Andrade MV, de Souza Noronha KVM, Kind P. EQ-5D-3L as a health measure of Brazilian adult population. Qual Life Res. 2015;24:2761-76. Medline:25896666 doi:10.1007/s11136-015-0994-7

27 Janssen MF, Szende A, Cabases J, Ramos-Goni JM, Vilagut G, Konig HH. Population norms for the EQ-5D-3L: a cross-country analysis of population surveys for 20 countries. Eur J Health Econ. 2019;20:205-16. Medline:29445941 doi:10.1007/ s10198-018-0955-5

28 Shiroiwa T, Ikeda S, Noto S, Igarashi A, Fukuda T, Saito S, et al. Comparison of value set based on DCE and/or TTO data: scoring for EQ-5D-5L health states in Japan. Value Health. 2016;19:648-54. Medline:27565282 doi:10.1016/j.jval.2016.03.1834

29 Liu GG, Wu H, Li M, Gao C, Luo N. Chinese time trade-off values for EQ-5D health states. Value Health. 2014;17:597-604. Medline:25128053 doi:10.1016/j.jval.2014.05.007

$30 \mathrm{Wu}$ C, Gong Y, Wu J, Zhang S, Yin X, Dong X, et al. Chinese Version of the EQ-5D Preference Weights: Applicability in a Chinese General Population. PLoS One. 2016;11:e0164334. Medline:27711169 doi:10.1371/journal.pone.0164334

31 Sun S, Chen J, Johannesson M, Kind P, Xu L, Zhang Y, et al. Population health status in China: EQ-5D results, by age, sex and socio-economic status, from the National Health Services Survey 2008. Qual Life Res. 2011;20:309-20. Medline:21042861 doi:10.1007/s11136-010-9762-x

32 Luo N, Liu G, Li M, Guan H, Jin X, Rand-Hendriksen K. Estimating an EQ-5D-5L value set for China. Value Health. 2017;20:6629. Medline:28408009 doi:10.1016/j.jval.2016.11.016

33 Wang H, Kindig DA, Mullahy J. Variation in Chinese population health related quality of life: results from a EuroQol study in Beijing, China. Qual Life Res. 2005;14:119-32. Medline:15789946 doi:10.1007/s11136-004-0612-6

34 Wang H-M, Patrick DL, Edwards TC, Skalicky AM, Zeng H-Y, Gu W-W. Validation of the EQ-5D in a general population sample in urban China. Qual Life Res. 2012;21:155-60. Medline:21505881 doi:10.1007/s11136-011-9915-6

35 Zhang Y, Zhou Z, Gao J, Wang D, Zhang Q, Zhou Z, et al. Health-related quality of life and its influencing factors for patients with hypertension: evidence from the urban and rural areas of Shaanxi Province, China. BMC Health Serv Res. 2016;16:277. Medline:27430314 doi:10.1186/s12913-016-1536-x

36 Lin K, Yang X, Yin G, Lin S. Diabetes self-care activities and health-related quality-of-life of individuals with type 1 diabetes mellitus in Shantou, China. J Int Med Res. 2016;44:147-56. Medline:26658458 doi:10.1177/0300060515597933

37 Lee CF, Ng R, Luo N, Wong NS, Yap YS, Lo SK, et al. The English and Chinese versions of the five-level EuroQoL Group's five-dimension questionnaire (EQ-5D) were valid and reliable and provided comparable scores in Asian breast cancer patients. Support Care Cancer. 2013;21:201-9. Medline:22669608 doi:10.1007/s00520-012-1512-X

38 Sun S, Chen J, Kind P, Xu L, Zhang Y, Burström K. Experience-based VAS values for EQ-5D-3L health states in a national general population health survey in China. Qual Life Res. 2015;24:693-703. Medline:25246184 doi:10.1007/s11136-014-0793-6

39 Huang W, Yu H, Liu C, Liu G, Wu Q, Zhou J, et al. Assessing health-related quality of life of chinese adults in heilongjiang using EQ-5D-3L. Int J Environ Res Public Health. 2017;14:224. Medline:28241507 doi:10.3390/ijerph14030224

$40 \mathrm{Wu}$ H, Liu G, Guan H. Analysis on Influential Factors of Health Utility Evaluation. China Pharmacy. 2016;27:1450-3.

41 Zhuo L, Xu L, Ye J, Sun S, Zhang Y, Burstrom K, et al. Time Trade-Off Value Set for EQ-5D-3L Based on a Nationally Representative Chinese Population Survey. Value Health. 2018;21:1330-7. Medline:30442281 doi:10.1016/j.jval.2018.04.1370

42 Center for Health Statistics and Information MoHC. An Analysis Report of National Health Services Survey in China, 2013. Beijing: Center for Health Statistics and Information, Ministry of Health China, 2015.

43 Feng XL, Xu L, Guo Y, Ronsmans C. Socioeconomic inequalities in hospital births in China between 1988 and 2008 . Bull World Health Organ. 2011;89:432-41. Medline:21673859 doi:10.2471/BLT.10.085274 
44 Sun S, Chen J, Johannesson M, Kind P, Xu L, Zhang Y, et al. Regional differences in health status in China: Population health-related quality of life results from the National Health Services Survey 2008. Health Place. 2011;17:671-80. Medline:21334961 doi:10.1016/j.healthplace.2011.01.007

45 Meng Q, Xu L, Zhang Y, Qian J, Cai M, Xin Y, et al. Trends in access to health services and financial protection in China between 2003 and 2011: a cross-sectional study. Lancet. 2012;379:805-14. Medline:22386034 doi:10.1016/S0140-6736(12)60278-5

46 Zhou ZL, Fang Y, Zhou ZY, Li D, Wang D, Li YL, et al. Assessing Income-Related Health Inequality and Horizontal Inequity in China. Soc Indic Res. 2017;132:241-56. doi:10.1007/s11205-015-1221-1

47 Garcia-Gordillo MA, Adsuar J, Olivares P. Normative values of EQ-5D-5L: in a Spanish representative population sample from Spanish Health Survey, 2011. Qual Life Res. 2016;25:1313-21. Medline:26482825 doi:10.1007/s11136-015-1164-7

48 EuroQol Research Foundation. EQ-5D-3L User Guide: Basic information on how to use the EQ-5D-3L instrument. 2018. Available: https://euroqol.org/publications/user-guides/. Accessed: 25 January 2021.

49 Organization WH. Social determinants of health. WHO Regional Office for South-East Asia, 2008.

50 Amemiya T. Tobit models: A survey. J Econom. 1984;24:3-61. doi:10.1016/0304-4076(84)90074-5

51 Cohen J. Statistical power analysis. Curr Dir Psychol. 1992;1:98-101. doi:10.1111/1467-8721.ep10768783

52 Norman GR, Sloan JA, Wyrwich KW. Interpretation of changes in health-related quality of life: the remarkable universality of half a standard deviation. Med Care. 2003;41:582-92. Medline:12719681 doi:10.1097/01.MLR.0000062554.74615.4C

53 National Bureau of Statistics of China. Statistical Communiqué of the People's Republic of China on the National Economic and Social Development 2017. Available: http://www.stats.gov.cn/tjsj/ndsj/2017/indexch.htm. Accessed: 25 January 2021.

54 Lee YK, Nam HS, Chuang LH, Kim KY, Yang HK, Kwon IS, et al. South Korean time trade-off values for EQ-5D health states: modeling with observed values for 101 health states. Value Health. 2009;12:1187-93. Medline:19659703 doi:10.1111/j.15244733.2009.00579.x

55 Yao Q, Zhang Y, Xu L. Factors Affect Chinese Health-Related Quality of Life and the Effectiveness of Healthcare Reforms. Pop Develop. 2018;24:85-95.

56 Cheung YB, Thumboo J. Developing health-related quality-of-life instruments for use in Asia. Pharmacoeconomics. 2006;24:64350. Medline:16802840 doi:10.2165/00019053-200624070-00003

$57 \mathrm{Wu}$ Y, Xie G. Development and assessment of Chinese general quality of life instrument. Handbook of disease burdens and quality of life measures. 2010:265-83.

58 Björk S, Norinder A. The weighting exercise for the Swedish version of the EuroQol. Health Econ. 1999;8:117-26. Medline:10342725 doi:10.1002/(SICI)1099-1050(199903)8:2<117::AID-HEC402>3.0.CO;2-A

59 Devlin NJ, Hansen P, Kind P, Williams AH. The health state preferences and logical inconsistencies of New Zealanders: a tale of two tariffs. Discussion paper-university of york centre for health economics. 2000.

60 Abdin E, Subramaniam M, Vaingankar JA, Luo N, Chong SA. Measuring health-related quality of life among adults in Singapore: population norms for the EQ-5D. Qual Life Res. 2013;22:2983-91. Medline:23549857 doi:10.1007/s11136-013-0405-X

61 Yao Q, Liu C, Zhang Y, Xu L. Changes in health-related quality of life of Chinese populations measured by the EQ-5D-3 L: a comparison of the 2008 and 2013 National Health Services Surveys. Health Qual Life Outcomes. 2019;17:43. Medline:30866953 doi:10.1186/s12955-019-1109-x

62 Ebrahim S, Parshuram C. Comparison of utility scores from the Visual Analog Scale and Health Utilities Index 3 in children following pediatric intensive care unit admission. J Child Health Care. 2015;19:53-62. Medline:23939724 doi:10.1177/1367493513496909

63 Zhang T, Shi W, Huang Z, Gao D, Guo Z, Liu J, et al. Influence of culture, residential segregation and socioeconomic development on rural elderly health-related quality of life in Guangxi, China. Health Qual Life Outcomes. 2016;14:98. Medline:27356505 doi:10.1186/s12955-016-0499-2

64 Liang Y, Wu W. Exploratory analysis of health-related quality of life among the empty-nest elderly in rural China: An empirical study in three economically developed cities in eastern China. Health Qual Life Outcomes. 2014;12:59. Medline:24766880 doi:10.1186/1477-7525-12-59

65 Lei P, Xu L, Nwaru B, Long Q, Wu Z. Social networks and health-related quality of life among Chinese old adults in urban areas: results from 4th National Household Health Survey. Public Health. 2016;131:27-39. Medline:26631913 doi:10.1016/j.puhe.2015.10.009

66 Tan Z, Liang Y, Liu S, Cao W, Tu H, Guo L, et al. Health-related quality of life as measured with EQ-5D among populations with and without specific chronic conditions: a population-based survey in Shaanxi Province, China. PLoS One. 2013;8:e65958. Medline:23843948 doi:10.1371/journal.pone.0065958

67 Cui J, Tang J, Qin J, Zhang C, Chen Y. Self-evaluated Health Condition and Its Influencing Factors of Residents in Hami Area. Chin Gen Prac. 2015;8:23.

68 Wang X, Guo H, Chen J. An empirical study on self-rated health status and reporting behaviors of rural residents in China. Zhongguo Weisheng Zhengce Yanjiu. 2016;9:68-74.

69 Janssen MF, Bonsel GJ, Luo N. Is EQ-5D-5L better than EQ-5D-3L? A head-to-head comparison of descriptive systems and value sets from seven countries. Pharmacoeconomics. 2018;36:675-97. Medline:29470821 doi:10.1007/s40273-018-0623-8

70 Zhou T, Guan H, Liu G, Ma A. Comparasion Analysis on EQ-5D-3L and EQ-5D-5Lfor General Population in China. Zhongguo Weisheng Jingji. 2016;35:17-20.

71 Selivanova A, Buskens E, Krabbe PF. Head-to-Head Comparison of EQ-5D-3L and EQ-5D-5L Health Values. Pharmacoeconomics. 2018;36:715-25. Medline:29623559 doi:10.1007/s40273-018-0647-0

72 Kularatna S, Senanayake S, Gunawardena N, Graves N. Comparison of the EQ-5D 3L and the SF-6D (SF-36) contemporaneous utility scores in patients with chronic kidney disease in Sri Lanka: a cross-sectional survey. BMJ Open. 2019;9:e024854. Medline:30772857 doi:10.1136/bmjopen-2018-024854

73 Ferreira LN, Ferreira PL, Ribeiro FP, Pereira LN. Comparing the performance of the EQ-5D-3L and the EQ-5D-5L in young Portuguese adults. Health Qual Life Outcomes. 2016;14:89. Medline:27267761 doi:10.1186/s12955-016-0491-X 